\title{
ON B-SPLINE FRAMELETS DERIVED FROM THE UNITARY EXTENSION PRINCIPLE
}

\author{
ZUOWEI SHEN AND ZHIQIANG XU
}

\begin{abstract}
Spline wavelet tight frames of 20] have been used widely in frame based image analysis and restorations (see, e.g. survey articles [15, 22 ). However, except for the tight frame property and the approximation order of the truncated series (see [13, 20]), there are few other properties of this family of spline wavelet tight frames to be known. This paper is to present a few new properties of this family that will provide further understanding of it and, hopefully, give some indications why it is efficient in image analysis and restorations. In particular, we present a recurrence formula of computing generators of higher order spline wavelet tight frames from the lower order ones. We also represent each generator of spline wavelet tight frames as certain order of derivative of some univariate box spline. With this, we further show that each generator of sufficiently high order spline wavelet tight frames is close to a right order of derivative of a properly scaled Gaussian function. This leads to the result that the wavelet system generated by a finitely many consecutive derivatives of a properly scaled Gaussian function forms a frame whose frame bounds can be almost tight.
\end{abstract}

\section{INTRODUCTION}

This paper is to investigate the family of the spline wavelet tight frames derived from [20]. We start with basic notions. For given $\Psi:=\left\{\psi_{1}, \ldots, \psi_{r}\right\} \subset$ $L_{2}(\mathbb{R})$, the wavelet system generated by $\Psi$ is defined as

$$
X(\Psi):=\left\{\psi_{\ell, n, k}:=2^{n / 2} \psi_{\ell}\left(2^{n} \cdot-k\right): 1 \leq \ell \leq r ; n, k \in \mathbb{Z}\right\} .
$$

The system $X(\Psi) \subset L_{2}(\mathbb{R})$ is called a tight frame if

$$
f=\sum_{g \in X(\Psi)}\langle f, g\rangle g
$$

holds for all $f \in L_{2}(\mathbb{R})$. If $X(\Psi) \subset L_{2}(\mathbb{R})$ is a tight frame system of $L_{2}(\mathbb{R})$ generated by a Multiresolution analysis (MRA), then its generators $\Psi$ are called as a framelet.

Zuowei Shen was supported by R146-000-113-112 from the National University of Singapore. Zhiqiang Xu was supported by NSFC grant 11171336 and by the Funds for Creative Research Groups of China (Grant No. 11021101). 
The MRA starts from a refinable function $\varphi$. A compactly supported function $\varphi$ is refinable if it satisfies a refinement equation

$$
\varphi(x)=2 \sum_{j \in \mathbb{Z}} a_{j} \varphi(2 x-j),
$$

for some sequence $a \in \ell_{2}(\mathbb{Z})$. Refinable equation (11) can be written via its Fourier transform as

$$
\widehat{\varphi}(\omega)=\widehat{a}\left(\frac{\omega}{2}\right) \cdot \widehat{\varphi}\left(\frac{\omega}{2}\right), \quad \text { a.e. } \quad \omega \in \mathbb{R} .
$$

We call the sequence $a$ the refinement mask of $\varphi$ and $\widehat{a}(\omega)$ the refinement symbol of $\varphi$. Here, we use $\widehat{f}$ to denote the Fourier transform of $f \in L_{1}(\mathbb{R})$, which is defined as

$$
\widehat{f}(\omega):=\int_{-\infty}^{\infty} f(x) \exp (-i \omega x) d x .
$$

For a refinable function $\varphi \in L_{2}(\mathbb{R})$, let $V_{0}$ be the closed shift invariant space generated by $\{\varphi(\cdot-k): k \in \mathbb{Z}\}$ and $V_{j}:=\left\{f\left(2^{j} \cdot\right): f \in V_{0}\right\}, j \in \mathbb{Z}$. It is known that when $\varphi$ is compactly supported, then $\left\{V_{j}\right\}_{j \in \mathbb{Z}}$ forms a multiresolution analysis. Recall that a multiresolution analysis is a family of closed subspaces $\left\{V_{j}\right\}_{j \in \mathbb{Z}}$ of $L_{2}(\mathbb{R})$ that satisfies: (i) $V_{j} \subset V_{j+1}$, (ii) $\bigcup_{j} V_{j}$ is dense in $L_{2}(\mathbb{R})$ and (iii) $\bigcap_{j} V_{j}=\{0\}$ (see [2] and [19]).

A special family of refinable functions is B-splines. Let $\varphi^{(m)}$ be the centered B-spline of order $m$, which is defined in Fourier domain by

$$
\hat{\varphi}^{(m)}(\omega)=e^{-\frac{i \omega j_{m}}{2}} \operatorname{sinc}\left(\frac{\omega}{2}\right)^{m}
$$

where

(3) $\quad \operatorname{sinc}(x):=\left\{\begin{array}{ll}\sin (x) / x, & \text { for } x \neq 0 \\ 1, & \text { for } x=0\end{array} ;\right.$ and $\quad j_{m}:=\left\{\begin{array}{ll}0, & m \text { is even } \\ 1, & m \text { is odd }\end{array}\right.$.

Then $\varphi^{(m)}$ is refinable with refinement symbol

$$
\hat{a}^{(m)}(\omega)=e^{-\frac{i \omega j_{m}}{2}} \cos ^{m}\left(\frac{\omega}{2}\right) .
$$

The tight framelets can be constructed by the unitary extension principle (UEP) of [20] from a given multiresolution analysis. For a given B-spline $\varphi^{(m)}$ of order $m$, it was shown in [20] that the $m$ functions, $\Psi^{(m)}=\left\{\psi_{\ell}^{(m)}\right.$ : $\ell=1, \ldots, m\}$, defined in Fourier domain by

$$
\hat{\psi}_{\ell}^{(m)}(\omega):=i^{\ell} e^{-\frac{i \omega j_{m}}{2}} \sqrt{\left(\begin{array}{c}
m \\
\ell
\end{array}\right)} \frac{\cos ^{m-\ell}(\omega / 4) \sin ^{m+\ell}(\omega / 4)}{(\omega / 4)^{m}},
$$

form a tight wavelet frame in $L_{2}(\mathbb{R})$, i.e. $\Psi^{(m)}$ is a framelet set. We call $\Psi^{(m)}$ as the B-spline framelet of order $m$. The B-spline framelet is either symmetric or anti-symmetric and has small supports for a given smoothness order. Similar with B-splines, each B-spline framelet has an analytic form. 
Since the publication of the unitary extension principle (UEP) of [20] in 1997, there are many theoretic developments and applications of MRA based wavelet frames. In particular, the B-spline framelets $\Psi^{(m)}$ derived from the UEP in 20, are widely used in various applications, which include image inpainting in [5]; image denoising in [8]; high and super resolution image reconstruction in [10]; deblurring and blind debluring in [6] 9]; and image segmentation in [14. The interested reader should consult the survey articles [15,22 for details.

The paper is organized as follows: Section 2, which contains two subsections, is for some basic properties of B-spline framelets. In particular, in sub-section 2.1, we present recurrence formulas for B-spline framelets $\psi_{\ell}^{(m)}$, in which the well-known recurrence formula of B-splines can be viewed as a special form of recurrence formulas of B-spline framelets. This gives a fast algorithm for computing them. We further show that the B-spline framelets can be derived from the $\ell$ th derivative of some univariate box spline in subsection 2.2, This was implicity used in 4, to approximate the some derivatives of a function. In Section 3, we investigate the asymptotic property of B-spline framlets $\psi_{\ell}^{(m)}, \ell=1, \ldots, m$. We firstly prove that the univariate box splines defined in Section 2 uniformly converge to a Gaussian function under a mild condition, and we further show that

$$
\max _{1 \leq \ell \leq m} \max _{x \in \mathbb{R}}\left|\psi_{\ell}^{(m)}(x)-G_{\ell}^{(m)}(x)\right| \lesssim \frac{(\ln m)^{5 / 2}}{m^{3 / 2}},
$$

where $G_{\ell}^{(m)}$ is the $\ell$ th derivative of some scaled Gaussian function $G(x)$ (see Section 3.2 for the detailed definition).

This leads to discover that wavelet system generated by a finite number of consecutive directives of scaled Gaussian function form a frame whose bounds are almost tight, and that is done in Section 4.

\section{Properties of B-Spline framelets}

In this section, we give a recurrence formula of the B-spline framelets which computes higher order framelets from lower order ones. We also show that one can represent the derivatives of higher order framelets by lower order framelets. Furthermore, we derive another set of formulas that represents each framelet as a derivative of a univariate box spline which is already implicitly used in [4], where a theory is developed to connect the PDE based and spline wavelet based image restorations.

2.1. Recurrence formulas of B-spline framelets. While the recurrence formulas for B-splines and their derivatives are well-known (see [1]), the corresponding formulas for B-spline framelets are not available yet. This section is to establish such formulas. Let $B_{m}:=\varphi^{(m)}\left(\cdot+j_{m} / 2\right)$, where $\varphi^{(m)}$ is given in (2) and $j_{m}$ is defined (3). Recall the following well-known 
recurrence formula of B-splines:

$$
B_{m+1}(x)=\frac{2 x+m+1}{2 m} B_{m}\left(x+\frac{1}{2}\right)+\frac{m+1-2 x}{2 m} B_{m}\left(x-\frac{1}{2}\right) .
$$

Based on (5), one can compute B-splines fast and easily which makes Bsplines useful. The derivative of B-splines can be computed by the lower order splines as given below:

$$
\frac{d}{d x} B_{m+1}(x)=B_{m}\left(x+\frac{1}{2}\right)-B_{m}\left(x-\frac{1}{2}\right) .
$$

The aim of this section is to give the corresponding formulas for the B-spline framelets $\psi_{\ell}^{(m)}, \ell=1, \ldots, m$. To state the formulas conveniently, we present the formulas for the function $\tilde{\psi}_{\ell}^{(m)}(\cdot):=\psi_{\ell}^{(m)}\left(\cdot+\frac{j_{m}}{2}\right)$. Note that the Fourier transform of $\tilde{\psi}_{\ell}^{(m)}$ is

$$
\widehat{\tilde{\psi}}_{\ell}^{(m)}(\omega)=i^{\ell} \sqrt{\left(\begin{array}{c}
m \\
\ell
\end{array}\right)} \frac{\cos ^{m-\ell}\left(\frac{\omega}{4}\right) \sin ^{m+\ell}\left(\frac{\omega}{4}\right)}{\left(\frac{\omega}{4}\right)^{m}} .
$$

We note that the formulas presented in this subsection are used to calculate the function value and the derivative of $\tilde{\psi}_{\ell}^{(m)}$. When $m$ is even, $\psi_{\ell}^{(m)} \equiv \tilde{\psi}_{\ell}^{(m)}$. When $m$ is odd, one can obtain those of the function $\psi_{\ell}^{(m)}$ by the halftranslation of $\tilde{\psi}_{\ell}^{(m)}$. Hence, the formulas given in this subsection also work for $\psi_{\ell}^{(m)}$ with a proper shift.

Next, we present the recurrence relations of framelets $\tilde{\psi}_{\ell}^{(m)}$.

Theorem 1. Let $m \in \mathbb{N}$ be given and $1 \leq \ell \leq m$ and let the framelet $\tilde{\psi}_{\ell}^{(m)}$ derived from B-spline of order $m$ be given via its Fourier transform as (7). Then, we have the following recurrence formula between $\tilde{\psi}_{\ell}^{(m+1)}$ and $\tilde{\psi}_{\ell}^{(m)}$ : for $1 \leq \ell \leq m$ :

$$
\text { (8) } \begin{aligned}
\tilde{\psi}_{\ell}^{(m+1)}(x)=\sqrt{\frac{m+1}{m+1-\ell}} \\
\left(\frac{2 x+m+1}{2 m} \tilde{\psi}_{\ell}^{(m)}\left(x+\frac{1}{2}\right)+\frac{m+1-2 x}{2 m} \tilde{\psi}_{\ell}^{(m)}\left(x-\frac{1}{2}\right)+\frac{\ell}{m} \tilde{\psi}_{\ell}^{(m)}(x)\right) ;
\end{aligned}
$$

the recurrence formula between $\tilde{\psi}_{m+1}^{(m+1)}$ and $\tilde{\psi}_{m}^{(m)}$ is:

$$
\tilde{\psi}_{m+1}^{(m+1)}(x)=\frac{2 x+m+1}{2 m} \tilde{\psi}_{m}^{(m)}\left(x+\frac{1}{2}\right)+\frac{2 x-m-1}{2 m} \tilde{\psi}_{m}^{(m)}\left(x-\frac{1}{2}\right)-\frac{2 x}{m} \tilde{\psi}_{m}^{(m)}(x) .
$$

Proof. We firstly prove (8) which is done in Fourier domain. Note that

$$
\frac{d}{d \omega} \widehat{\widetilde{\psi}}_{\ell}^{(m)}(\omega)=-i \int_{-\infty}^{\infty} x \tilde{\psi}_{\ell}^{(m)}(x) e^{-i \omega x} d x
$$


which implies that the Fourier transform of function $g_{\ell}(x):=x \tilde{\psi}_{\ell}^{(m)}(x)$ is (10)

$$
\hat{g}_{\ell}(\omega)=i^{\ell+1} \cdot 4^{m-1} \cdot \sqrt{\left(\begin{array}{c}
m \\
\ell
\end{array}\right)} \cos \left(\frac{\omega}{4}\right)^{m-\ell-1} \sin \left(\frac{\omega}{4}\right)^{m+\ell-1} \frac{m \omega \cos \left(\frac{\omega}{2}\right)-2 m \sin \left(\frac{\omega}{2}\right)+\ell \cdot \omega}{\omega^{m+1}} .
$$

Note that

$$
\frac{2 x+m+1}{2 m} \tilde{\psi}_{\ell}^{(m)}\left(x+\frac{1}{2}\right)=\frac{1}{m} g_{\ell}\left(x+\frac{1}{2}\right)+\frac{1}{2} \tilde{\psi}_{\ell}^{(m)}\left(x+\frac{1}{2}\right)
$$

and

$$
\frac{m+1-2 x}{2 m} \tilde{\psi}_{\ell}^{(m)}\left(x-\frac{1}{2}\right)=\frac{1}{2} \tilde{\psi}_{\ell}^{(m)}\left(x-\frac{1}{2}\right)-\frac{1}{m} g_{\ell}\left(x-\frac{1}{2}\right) .
$$

A simple manipulation shows that the Fourier transform of the right hand side of (8) becomes

$$
\begin{aligned}
& \sqrt{\frac{m+1}{m+1-\ell}}\left(\frac{1}{m} \exp \left(\frac{i \omega}{2}\right) \hat{g}_{\ell}(\omega)+\frac{1}{2} \cdot\left(\exp \left(\frac{i \omega}{2}\right)+\exp \left(-\frac{i \omega}{2}\right)\right) \widehat{\tilde{\psi}}_{\ell}^{(m)}(\omega)\right. \\
& \left.-\frac{1}{m} \exp \left(-\frac{i \omega}{2}\right) \hat{g}_{\ell}(\omega)+\frac{\ell}{m} \widehat{\tilde{\psi}}_{\ell}^{(m)}(\omega)\right) \\
= & i^{\ell} \sqrt{\left(\begin{array}{c}
m+1 \\
\ell
\end{array}\right)} \frac{\cos ^{m+1-\ell}\left(\frac{\omega}{4}\right) \sin ^{m+1+\ell}\left(\frac{\omega}{4}\right)}{\left(\frac{\omega}{4}\right)^{m+1}}=\widehat{\tilde{\psi}}_{\ell}^{(m+1)}(\omega) .
\end{aligned}
$$

This proves (8). Similarly, the Fourier transform of the right side of (9) is

$$
\begin{aligned}
& \frac{1}{m}\left(\exp \left(\frac{i \omega}{2}\right)\left(\hat{g}_{m}(\omega)+\frac{m}{2} \widehat{\tilde{\psi}}_{m}^{(m)}(\omega)\right)+\exp \left(-\frac{i \omega}{2}\right)\left(\hat{g}_{m}(\omega)-\frac{m}{2} \widehat{\widetilde{\psi}}_{m}^{(m)}(\omega)\right)-2 \hat{g}_{m}(\omega)\right) \\
= & i^{m+1} \frac{\sin ^{2 m+2}\left(\frac{\omega}{4}\right)}{\left(\frac{\omega}{4}\right)^{m+1}}=\widehat{\tilde{\psi}}_{m+1}^{(m+1)}(\omega),
\end{aligned}
$$

which proves (9).

Furthermore, combining (8) and (9), we have a recurrence algorithm for efficiently computing $\tilde{\psi}_{\ell}^{(m)}, \ell=1, \ldots, m$. When $\ell<m$, we can use (8) to compute $\tilde{\psi}_{\ell}^{(m)}$ by $\tilde{\psi}_{\ell}^{(m-1)}$; we can use (9) to compute $\tilde{\psi}_{\ell}^{(\ell)}$ by $\tilde{\psi}_{\ell-1}^{(\ell-1)}$. Hence, we finally can reduce the computation of $\tilde{\psi}_{\ell}^{(m)}$ to that of $\tilde{\psi}_{1}^{(1)}$. Note that the function $\tilde{\psi}_{1}^{(1)}$ is Haar wavelet and

$$
\tilde{\psi}_{1}^{(1)}(x)= \begin{cases}1, & \text { if } x \in[-1 / 2,0) \\ -1, & \text { if } x \in[0,1 / 2] \\ 0, & \text { if }|x|>1 / 2\end{cases}
$$

We next show the method for computing $\tilde{\psi}_{2}^{(4)}$ by a table. In the following table, for the notation $\rightarrow$, we use the formula (8), while for the notation $\searrow$, we use (9): 


$$
\begin{aligned}
& \begin{array}{llll}
B_{1} & B_{2} & B_{3} & B_{4}
\end{array} \\
& \tilde{\psi}_{1}^{(1)} \quad \tilde{\psi}_{1}^{(2)} \quad \tilde{\psi}_{1}^{(3)} \quad \tilde{\psi}_{1}^{(4)} \\
& \searrow \tilde{\psi}_{2}^{(2)} \rightarrow \underset{\tilde{\psi}_{3}^{(3)}}{\tilde{\psi}_{3}^{(3)}} \rightarrow \underset{\tilde{\psi}_{3}^{(4)}}{\tilde{\psi}_{3}^{(4)}} . \\
& \tilde{\psi}_{4}^{(4)}
\end{aligned}
$$

Using the method, we compute $B_{5}$ and corresponding framelets (See Figure 1).
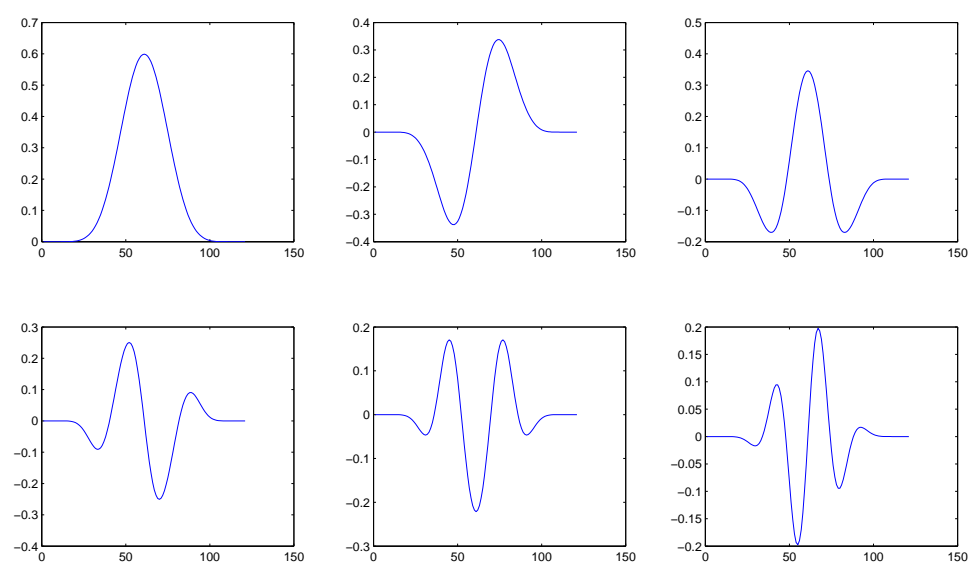

Figure 1. The $B_{5}$ and corresponding framelets.

Next, we give the recurrence formula for the derivatives of $\tilde{\psi}_{\ell}^{(m)}$ :

Theorem 2. Let $m \in \mathbb{N}$ be given and $1 \leq \ell \leq m$, and let the framelet $\tilde{\psi}_{\ell}^{(m)}$ derived from B-spline of order $m$ be defined by its Fourier transform as (7). When $1 \leq \ell \leq m-1$, we have

$$
\frac{d}{d x} \tilde{\psi}_{\ell}^{(m)}(x)=\sqrt{\frac{m}{m-\ell}}\left(\tilde{\psi}_{\ell}^{(m-1)}\left(x+\frac{1}{2}\right)-\tilde{\psi}_{\ell}^{(m-1)}\left(x-\frac{1}{2}\right)\right) .
$$

When $\ell=m$, we have

$$
\frac{d}{d x} \tilde{\psi}_{m}^{(m)}(x)=\tilde{\psi}_{m-1}^{(m-1)}\left(x+\frac{1}{2}\right)+\tilde{\psi}_{m-1}^{(m-1)}\left(x-\frac{1}{2}\right)-2 \tilde{\psi}_{m-1}^{(m-1)}(x) .
$$

Proof. We prove (11) here while (12) can be proven similarly. A simple calculation shows that the Fourier transform of the right side of (11) is

$$
\begin{aligned}
& \sqrt{\frac{m}{m-\ell}} \cdot \sqrt{\left(\begin{array}{c}
m-1 \\
\ell
\end{array}\right)} \cdot i^{\ell} \cdot \frac{\cos ^{m-1-\ell}\left(\frac{\omega}{4}\right) \sin ^{m-1+\ell}\left(\frac{\omega}{4}\right)}{\left(\frac{\omega}{4}\right)^{m-1}}\left(e^{i \frac{\omega}{2}}-e^{-i \frac{\omega}{2}}\right) \\
& =4 i^{\ell+1} \sqrt{\left(\begin{array}{c}
m \\
\ell
\end{array}\right)} \frac{\cos ^{m-\ell}\left(\frac{\omega}{4}\right) \sin ^{m+\ell}\left(\frac{\omega}{4}\right)}{\left(\frac{\omega}{4}\right)^{m-1}} .
\end{aligned}
$$


Note that the Fourier transform of $\frac{d}{d x} \tilde{\psi}_{\ell}^{(m)}(x)$ is

$$
4 \cdot i^{\ell+1} \cdot \sqrt{\left(\begin{array}{c}
m \\
\ell
\end{array}\right)} \frac{\cos ^{m-\ell}\left(\frac{\omega}{4}\right) \sin ^{m+\ell}\left(\frac{\omega}{4}\right)}{\left(\frac{\omega}{4}\right)^{m-1}} .
$$

Combining (13) and (14), we conclude (11).

Remark 1. Note that $\tilde{\psi}_{0}^{(m)}=B_{m}$. If we take $\ell=0$ in (8) , the recurrence relation (86) is reduced to (5), which is the recurrence formula for B-splines. Similarly, if we take $\ell=0$ in (11), then (11) is reduced to the derivative formula of B-splines (6).

2.2. Representing $\psi_{\ell}^{(m)}$ as the $\ell$ th derivative of a univariate box spline. We first recall the definition of box splines. The box spline $B(\cdot \mid \Xi)$ associated with a matrix $\Xi \in \mathbb{R}^{s \times n}$ is the distribution given by the rule (see [3])

$$
\int_{\mathbb{R}^{s}} B(x \mid \Xi) \varphi(x) d x=\int_{\left[-\frac{1}{2}, \frac{1}{2}\right)^{n}} \varphi(\Xi u) d u, \text { for all } \varphi \in \mathscr{D}\left(\mathbb{R}^{s}\right),
$$

where $\mathscr{D}\left(\mathbb{R}^{s}\right)$ is the test function space. The box spline can be consider as a volume function of the section of unit cubes (see [3, 24, 25]). If we take $\Xi=(1,1, \ldots, 1) \in \mathbb{R}^{1 \times m}$, then the box spline $B(\cdot \mid \Xi)$ is reduced to a B-spline of order $m$. In the following theorem, we show that the B-spline framelet can be considered as the higher order derivative of a box spline up to a constant.

Theorem 3. Let $m \in \mathbb{N}$ be given and $1 \leq \ell \leq m$. Suppose that the framelet $\psi_{\ell}^{(m)}$ is defined by its Fourier transform in (4). Set

$$
\Xi_{m, \ell}:=[\underbrace{1, \ldots, 1}_{m-\ell}, \underbrace{\frac{1}{2}, \ldots, \frac{1}{2}}_{2 \ell}] .
$$

Then

$$
\psi_{\ell}^{(m)}(x)=\sqrt{\left(\begin{array}{c}
m \\
\ell
\end{array}\right)} \cdot \frac{1}{4^{\ell}} \cdot \frac{d^{\ell}}{d x^{\ell}} B\left(x-\left.\frac{j_{m}}{2}\right|_{m, \ell}\right),
$$

where $j_{m}$ is defined in (3). In particular, $\psi_{m}^{(m)}$ is the m-order derivative of $B_{2 m}\left(2 \cdot-j_{m}\right) / 4^{m}$, where $B_{2 m}$ is the B-spline of order $2 m$.

Proof. This again is proven in Fourier domain. It follows from the definition of box splines (15) that the Fourier transform of the box spline $B\left(\cdot \mid \Xi_{m, \ell}\right)$ is:

$$
\hat{B}\left(\omega \mid \Xi_{m, \ell}\right)=\left(\frac{\sin \frac{\omega}{2}}{\frac{\omega}{2}}\right)^{m-\ell}\left(\frac{\sin \frac{\omega}{4}}{\frac{\omega}{4}}\right)^{2 \ell} .
$$

Then the Fourier transform of

$$
\sqrt{\left(\begin{array}{c}
m \\
\ell
\end{array}\right)} \cdot \frac{1}{4^{\ell}} \cdot \frac{d^{\ell}}{d x^{\ell}} B\left(x-\frac{j_{m}}{2} \mid \Xi_{m, \ell}\right)
$$


can be computed as:

$$
\begin{aligned}
& \sqrt{\left(\begin{array}{c}
m \\
\ell
\end{array}\right)} \cdot \frac{1}{4^{\ell}} \cdot e^{-i j_{m} \omega / 2}(i \omega)^{\ell} \hat{B}\left(\omega \mid \Xi_{m, \ell}\right) \\
= & i^{\ell} \sqrt{\left(\begin{array}{c}
m \\
\ell
\end{array}\right)} e^{-i j_{m} \omega / 2}\left(\frac{\sin \frac{\omega}{2}}{\frac{\omega}{2}}\right)^{m-\ell} \frac{\left(\sin \frac{\omega}{4}\right)^{2 \ell}}{\left(\frac{\omega}{4}\right)^{\ell}} \\
= & i^{\ell} \sqrt{\left(\begin{array}{c}
m \\
\ell
\end{array}\right)} e^{-i j_{m} \omega / 2}\left(\frac{\sin \frac{\omega}{4} \cos \frac{\omega}{4}}{\frac{\omega}{4}}\right)^{m-\ell} \frac{\left(\sin \frac{\omega}{4}\right)^{2 \ell}}{\left(\frac{\omega}{4}\right)^{\ell}} \\
= & i^{\ell} \sqrt{\left(\begin{array}{c}
m \\
\ell
\end{array}\right)} e^{-i j_{m} \omega / 2} \frac{\cos ^{m-\ell}\left(\frac{\omega}{4}\right) \sin ^{m+\ell}\left(\frac{\omega}{4}\right)}{\left(\frac{\omega}{4}\right)^{m}} \\
= & \hat{\psi}_{\ell}^{(m)}(\omega),
\end{aligned}
$$

which proves (16). According to the definition of box splines, we have

$$
B_{2 m}\left(2 x-j_{m}\right)=B\left(x-\frac{j_{m}}{2} \mid \Xi_{m, m}\right) .
$$

And hence, $\psi_{m}^{(m)}$ is the $m$-order derivative of $B_{2 m}\left(2 \cdot-j_{m}\right) / 4^{m}$.

Theorem 3 states that each framelet of vanishing moment order of $\ell$ is the $\ell$ th derivative of a univariate box spline whose support is the same as the framelet and whose Fourier transform dose not vanish at origin. This fact is used in [4] to discretize differential operators by using framelets. We illustrate here for the case where $m$ is even how the framelet coefficients can be viewed as the samples of a difference of a given function which can be used to approximate the derivative of this function when it is smooth. Recall that, if $f \in L_{2}(\mathbb{R})$, then

$$
f=\sum_{\ell=1}^{m} \sum_{k, n \in \mathbb{Z}}\left\langle f, \psi_{\ell, n, k}^{(m)}\right\rangle \psi_{\ell, n, k}^{(m)} .
$$

We next show that the coefficients $\left\langle f, \psi_{\ell, n, k}^{(m)}\right\rangle$ is the $\ell$-order difference of a discretization of $f$ up to a constant. We set

$$
T_{n} f(x):=2^{\frac{n}{2}}\left\langle f\left(\frac{x+\cdot}{2^{n}}\right), B\left(\cdot \mid \Xi_{m, \ell}^{\prime}\right)\right\rangle,
$$

where

$$
\Xi_{m, \ell}^{\prime}:=[\underbrace{1, \ldots, 1}_{m-\ell}, \underbrace{\frac{1}{2}, \ldots, \frac{1}{2}}_{\ell}] .
$$

Then $T_{n} f$ can be considered as a discretization of $f$. We define the difference operator by

$$
\Delta_{\frac{1}{4}} T_{n} f(x):=T_{n} f\left(x+\frac{1}{4}\right)-T_{n} f\left(x-\frac{1}{4}\right) .
$$


Now we can give an explanation of the coefficient of $\left\langle f, \psi_{\ell, n, k}^{(m)}\right\rangle$ by the difference operator $\Delta_{\frac{1}{4}}$. In fact, we have

$$
\begin{aligned}
& \left\langle f, \psi_{\ell, n, k}^{(m)}\right\rangle \\
= & (-1)^{\ell} \sqrt{\left(\begin{array}{c}
m \\
\ell
\end{array}\right)} \cdot \frac{1}{4^{\ell}} \cdot 2^{n / 2} \cdot \frac{1}{2^{n \ell}} \cdot\left\langle f^{(\ell)}\left(\frac{\cdot+k}{2^{n}}\right), B\left(\cdot \mid \Xi_{m, \ell}\right)\right\rangle \\
= & (-1)^{\ell} \sqrt{\left(\begin{array}{c}
m \\
\ell
\end{array}\right)} \cdot \frac{1}{4^{\ell}} \cdot 2^{n / 2} \cdot \frac{1}{2^{n \ell}} \cdot \int_{[-1 / 2,1 / 2)^{m+\ell}} f^{(\ell)}\left(\frac{\Xi_{m, \ell} u+k}{2^{n}}\right) d u \\
= & (-1)^{\ell} \sqrt{\left(\begin{array}{c}
m \\
\ell
\end{array}\right)} \cdot \frac{1}{2^{\ell}} \cdot \Delta_{\frac{1}{4}}^{\ell} T_{n} f(k) .
\end{aligned}
$$

Here, the first identity follows from the result of Theorem 3 and the integration by parts. The second relation is obtained by the definition of box splines.

Remark 2. Theorem 3 shows that one can obtain the B-spline framelet by calculating the derivative of box splines, which provides a new path to construct spline framelets. We hope to construct multivariate spline framelets by calculating the derivative of some relevant box splines in future work.

\section{The Asymptotic Property of B-Spline Framelets}

3.1. The asymptotic convergence of univariate box splines. Up to the normalization, the B-spline tends to Gaussian function pointwise and in all $L_{p}$ norms with $2 \leq p<+\infty$ as the order tends to infinity (see [23]). Motivated by the results, in this subsection, we investigate the asymptotic convergence of univariate box splines, which is helpful to understand the convergence of $\psi_{\ell}^{(m)}$, with $\psi_{\ell}^{(m)}$ being the $\ell$-order derivative of a box spline up to a constant.

To state the results conveniently, throughout the rest of this paper, we shall use the notation $X \lesssim a, b, \ldots Y$ to refer to the inequality $X \leq C \cdot Y$, where the constant $C$ may depend on $a, b, \ldots$, but no other variable. In the next theorem, we show that the normalized box splines converge uniformly to a Gaussian function.

Theorem 4. For each $k \in \mathbb{N}$, Let

$$
\Xi_{k}:=\left[a_{1}^{(k)}, \ldots, a_{k}^{(k)}\right] \in \mathbb{R}^{1 \times k},
$$

be a given a set of points with $a_{j}^{(k)}>0,, j=1, \ldots, k$. Let $B\left(\cdot \mid \Xi_{k}\right)$ be the box spline associate with $\Xi_{k}$. Assume that

$$
\left\|\Xi_{k}\right\|_{2}^{2}=\sigma^{2}+\epsilon_{k},
$$


with $\sigma \in \mathbb{R}$ is a fixed constant and $\lim _{k \rightarrow \infty} \epsilon_{k}=0$, and assume that

$$
c_{1} \leq \frac{\max _{1 \leq j \leq k} a_{j}^{(k)}}{\min _{1 \leq j \leq k} a_{j}^{(k)}} \leq c_{2}
$$

where $c_{1}$ and $c_{2}$ are fixed positive constants independent of $k$. Then,

$$
\max _{x}\left|\sqrt{\frac{6}{\pi \sigma^{2}}} \exp \left(-\frac{6 x^{2}}{\sigma^{2}}\right)-B\left(x \mid \Xi_{k}\right)\right| \lesssim c_{1}, c_{2} \frac{(\ln k)^{3}}{k}+\left|\epsilon_{k}\right| \cdot\left|\ln \left(\left|\epsilon_{k}\right|\right)\right| \cdot \ln (k) .
$$

In order to prove Theorem 4 , we need the following technical lemma about the Fourier transform of the box spline $B\left(\cdot \mid \Xi_{k}\right)$ :

Lemma 5. Under the conditions of Theorem 4.

$$
\max _{\omega}\left|f_{k}(\omega)-\exp \left(-\frac{(\sigma \omega)^{2}}{24}\right)\right| \lesssim c_{1}, c_{2} \frac{(\ln k)^{2}}{k}+\left|\epsilon_{k}\right| \cdot|\ln | \epsilon_{k}||,
$$

where

$$
f_{k}(\omega):=\hat{B}\left(\omega \mid \Xi_{k}\right)=\prod_{j=1}^{k} \operatorname{sinc}\left(\frac{a_{j}^{(k)} \omega}{2}\right) .
$$

Proof. Without loss of generality, we suppose that for each fixed $k$

$$
0<a_{1}^{(k)} \leq a_{2}^{(k)} \leq \cdots \leq a_{k}^{(k)} .
$$

Then (17) and (18) imply that

$$
\frac{1}{\sqrt{k}} \lesssim_{c_{1}, c_{2}} a_{1}^{(k)} \leq a_{k}^{(k)} \lesssim_{c_{1}, c_{2}} \frac{1}{\sqrt{k}}
$$

We firstly consider the case $|\omega| \geq \pi / a_{k}^{(k)}$. Note that $\operatorname{sinc}(\cdot)$ is a monotone decreasing function in $[0, \pi]$ and

$$
|\operatorname{sinc}(\omega)| \leq \frac{1}{\pi}, \quad \text { for }|\omega| \geq \pi
$$

Then, we have

$$
\begin{aligned}
& \max _{|\omega| \geq \pi / a_{k}^{(k)}}\left|f_{k}(\omega)\right|=\max _{|\omega| \geq \pi / a_{k}^{(k)}} \prod_{j=1}^{k}\left|\operatorname{sinc}\left(\frac{a_{j}^{(k)} \omega}{2}\right)\right| \\
& \leq \max \left\{\frac{1}{\pi^{k}},\left(\operatorname{sinc}\left(\frac{\pi}{2} \frac{a_{1}^{(k)}}{a_{k}^{(k)}}\right)\right)^{k}\right\} \lesssim_{c_{1}, c_{2}} \beta^{k},
\end{aligned}
$$

where $\beta<1$ is a positive constant. And hence, when $|\omega| \geq \pi / a_{k}^{(k)}$,

$$
\left|f_{k}(\omega)-\exp \left(-\frac{(\sigma \omega)^{2}}{24}\right)\right| \leq\left|f_{k}(\omega)\right|+\exp \left(-\frac{(\sigma \omega)^{2}}{24}\right) \lesssim c_{1}, c_{2} \frac{1}{k},
$$

which implies (20). 
We next consider the case where $|\omega| \leq \pi / a_{k}^{(k)}$. Taylor expansion shows that, when $|\omega| \leq \pi / a_{k}^{(k)}$,

$$
\ln f_{k}(\omega)=\sum_{j=1}^{k} \ln \left(\operatorname{sinc}\left(\frac{a_{j}^{(k)} \omega}{2}\right)\right)=-\left(\left\|\Xi_{k}\right\|_{2}^{2} \cdot \frac{\omega^{2}}{24}+S(\omega)\right),
$$

where

$$
S(\omega)=\frac{\left\|\Xi_{k}\right\|_{4}^{4} \cdot \omega^{4}}{2880}+\frac{\left\|\Xi_{k}\right\|_{6}^{6} \cdot \omega^{6}}{181440}+\cdots
$$

is a uniformly convergent series on $|\omega| \leq \pi / a_{k}^{(k)}$. By (22), we now obtain that, when $|\omega| \leq \pi / a_{k}^{(k)}$,

$$
f_{k}(\omega)=\prod_{j=1}^{k} \operatorname{sinc}\left(\frac{a_{j}^{(k)} \omega}{2}\right)=\exp \left(-\frac{(\sigma \omega)^{2}}{24}\right) \cdot \exp \left(-\frac{\epsilon_{k} \omega^{2}}{24}\right) \cdot \exp (-S(\omega)) .
$$

Hence,

$$
\begin{aligned}
& \left|f_{k}(\omega)-\exp \left(-\frac{\sigma^{2} \omega^{2}}{24}\right)\right| \\
\leq & \exp (-S(\omega)) \exp \left(-\frac{\sigma^{2} \omega^{2}}{24}\right) \cdot\left|\left(\exp \left(-\frac{\epsilon_{k} \omega^{2}}{24}\right)-1\right)\right| \\
& +\exp \left(-\frac{\sigma^{2} \omega^{2}}{24}\right) \cdot|\exp (-S(\omega))-1| .
\end{aligned}
$$

Once, we prove that

$$
\exp \left(-\frac{\sigma^{2} \omega^{2}}{24}\right) \cdot\left|\exp \left(-\frac{\epsilon_{k} \omega^{2}}{24}\right)-1\right| \lesssim\left|\epsilon_{k}\right| \cdot|\ln | \epsilon_{k}||
$$

and

$$
\exp \left(-\frac{\sigma^{2} \omega^{2}}{24}\right) \cdot|\exp (-S(\omega))-1| \lesssim \frac{(\ln k)^{2}}{k} .
$$

Then, combining (23), (24) and (25), we obtain (20).

It remains to prove (24) and (25). We first prove that

$$
\exp \left(-\frac{\sigma^{2} \omega^{2}}{24}\right) \cdot\left|\exp \left(-\frac{\epsilon_{k} \omega^{2}}{24}\right)-1\right| \lesssim\left|\epsilon_{k}\right| \cdot|\ln | \epsilon_{k}|| \cdot
$$

By Taylor's expansion, when $\omega^{2} \leq 24 \cdot|\ln | \epsilon_{k}|| / \sigma^{2}$,

$$
\exp \left(-\frac{\sigma^{2} \omega^{2}}{24}\right)\left|\exp \left(-\frac{\epsilon_{k} \omega^{2}}{24}\right)-1\right| \leq\left|\left(\exp \left(-\frac{\epsilon_{k} \omega^{2}}{24}\right)-1\right)\right| \lesssim\left|\epsilon_{k}\right| \cdot|\ln | \epsilon_{k}|| ;
$$

when $\omega^{2} \geq 24 \cdot|\ln | \epsilon_{k}|| / \sigma^{2}$,

$$
\exp \left(-\frac{\sigma^{2} \omega^{2}}{24}\right)\left|\exp \left(-\frac{\epsilon_{k} \omega^{2}}{24}\right)-1\right| \leq 2 \exp \left(-\frac{\sigma^{2} \omega^{2}}{24}\right) \lesssim\left|\epsilon_{k}\right| \text {. }
$$

This gives (24). 
We next prove that (25). Note that, when $|\omega| \leq \sqrt{24 \ln k} / \sigma$,

$$
\exp \left(-\frac{\sigma^{2} \omega^{2}}{24}\right)|1-\exp (-S(\omega))| \lesssim|1-\exp (-S(\omega))| \lesssim c_{1}, c_{2} \frac{(\ln k)^{2}}{k} .
$$

When $\sqrt{24 \ln k} / \sigma \leq|\omega| \leq \pi / a_{k}^{(k)}$, we have

$$
\exp \left(-\frac{\sigma^{2} \omega^{2}}{24}\right) \leq \frac{1}{k}
$$

which implies that

$$
\exp \left(-\frac{\sigma^{2} \omega^{2}}{24}\right)|\exp (-S(\omega))-1| \leq \frac{2}{k} \lesssim \frac{(\ln k)^{2}}{k} .
$$

Combining (26) and (27), one derives (25).

Proof of Theorem 4, Note that

$$
\begin{aligned}
\frac{1}{2 \pi} \int_{-\infty}^{\infty} \exp \left(-\frac{\sigma^{2} \omega^{2}}{24}\right) \exp (i \omega x) d \omega & =\sqrt{\frac{6}{\pi \sigma^{2}}} \exp \left(-\frac{6 x^{2}}{\sigma^{2}}\right), \\
\frac{1}{2 \pi} \int_{-\infty}^{\infty} f_{k}(\omega) \exp (i \omega x) d \omega & =B\left(x \mid \Xi_{k}\right) .
\end{aligned}
$$

Then

$$
\begin{aligned}
& \max _{x}\left|\sqrt{\frac{6}{\pi \sigma^{2}}} \exp \left(-\frac{6 x^{2}}{\sigma^{2}}\right)-B\left(x \mid \Xi_{k}\right)\right| \lesssim \int_{-\infty}^{\infty}\left|\exp \left(-\frac{\sigma^{2} \omega^{2}}{24}\right)-f_{k}(\omega)\right| d \omega \\
= & \int_{|\omega| \leq \frac{\sqrt{24} \ln k}{\sigma}}\left|\exp \left(-\frac{\sigma^{2} \omega^{2}}{24}\right)-f_{k}(\omega)\right| d \omega \\
+ & \int_{\frac{\sqrt{24} \ln k}{\sigma} \leq|\omega| \leq \frac{\pi}{a_{k}^{(k)}}}\left|\exp \left(-\frac{\sigma^{2} \omega^{2}}{24}\right)-f_{k}(\omega)\right| d \omega \\
+ & \int_{\frac{\pi}{a_{k}^{(k)}} \leq|\omega| \leq \frac{\pi}{a_{1}^{(k)}}}\left|\exp \left(-\frac{\sigma^{2} \omega^{2}}{24}\right)-f_{k}(\omega)\right| d \omega+\int_{|\omega| \geq \frac{\pi}{a_{1}^{(k)}}}\left|\exp \left(-\frac{\sigma^{2} \omega^{2}}{24}\right)-f_{k}(\omega)\right| d \omega \\
\lesssim & \frac{(\ln k)^{3}}{k}+\left|\epsilon_{k}\right| \cdot|\ln | \epsilon_{k}|| \cdot \ln k+\frac{\sqrt{k}}{k^{\ln k}}+\beta^{k} \lesssim \frac{(\ln k)^{3}}{k}+\left|\epsilon_{k}\right| \cdot|\ln | \epsilon_{k}|| \cdot \ln k,
\end{aligned}
$$

where $\beta=\max \left\{\frac{1}{\pi}, \operatorname{sinc}\left(\frac{\pi}{2} \frac{a_{1}^{(k)}}{a_{k}^{(k)}}\right)\right\}<1$. Here, we use (201) to obtain that

$$
\int_{|\omega| \leq \frac{\sqrt{24} \ln k}{\sigma}}\left|f_{k}(\omega)-\exp \left(-\frac{\sigma^{2} \omega^{2}}{24}\right)\right| d \omega \lesssim \frac{(\ln k)^{3}}{k}+\left|\epsilon_{k}\right| \cdot|\ln | \epsilon_{k}|| \cdot \ln k
$$

Note that $a_{k}^{(k)} / \sqrt{k}, k=1,2, \ldots$, is a bounded sequence and

$$
\exp \left(-\frac{\sigma^{2} \omega^{2}}{24}\right) \lesssim \frac{1}{k^{\ln k}}, \quad \text { for } \omega \geq \frac{\sqrt{24} \ln k}{\sigma} .
$$


Using a similar method as the proof of (27), we have that

$$
\int_{\frac{\sqrt{24} \ln k}{\sigma} \leq|\omega| \leq \frac{\pi}{a_{k}^{(k)}}}\left|f_{k}(\omega)-\exp \left(-\frac{\sigma^{2} \omega^{2}}{24}\right)\right| d \omega \lesssim \frac{\sqrt{k}}{k^{\ln k}} .
$$

To estimate

$$
\int_{|\omega| \geq \frac{\pi}{a_{1}^{(k)}}}\left|f_{k}(\omega)-\exp \left(-\omega^{2} / 24\right)\right| d \omega \leq \int_{|\omega| \geq \frac{\pi}{a_{1}^{(k)}}}\left|f_{k}(\omega)\right| d \omega+\int_{|\omega| \geq \frac{\pi}{a_{1}^{(k)}}} \exp \left(-\omega^{2} / 24\right) d \omega,
$$

we use the facts of

$$
\int_{|\omega| \geq \frac{\pi}{a_{1}^{(k)}}}\left|f_{k}(\omega)\right| d \omega \leq \int_{|\omega| \geq \frac{\pi}{a_{1}^{(k)}}}\left(\frac{2}{a_{1}^{(k)} \omega}\right)^{k} d \omega \lesssim \frac{1}{k}
$$

and

$$
\int_{|\omega| \geq \frac{\pi}{a_{1}^{(k)}}} \exp \left(-\frac{\omega^{2}}{24}\right) d \omega \lesssim \frac{1}{k}
$$

Theorem 4 implies that the normalized box spline $B\left(\cdot \mid \Xi_{m, \ell}\right)$ converges uniformly to a Gaussian function:

Corollary 6. Suppose that

$$
\Xi_{m, \ell}=[\underbrace{1, \ldots, 1}_{m-\ell}, \underbrace{1 / 2, \ldots, 1 / 2}_{2 \ell}] .
$$

Then, for each fixed $\ell, \sqrt{m-\frac{\ell}{2}} \cdot B\left(\sqrt{m-\frac{\ell}{2}} x \mid \Xi_{m, \ell}\right)$ converges uniformly to $\sqrt{\frac{6}{\pi}} \exp \left(-6 x^{2}\right)$, as $m \rightarrow \infty$.

Proof. By the definition of box splines, we have

$$
\sqrt{m-\frac{\ell}{2}} B\left(\sqrt{m-\frac{\ell}{2}} x \mid \Xi_{m, \ell}\right)=B\left(x \mid \frac{\Xi_{m, \ell}}{\sqrt{m-\ell / 2}}\right) .
$$

Note that, for each fixed $\ell,\left\|\frac{\Xi_{m, \ell}}{\sqrt{m-\ell / 2}}\right\|_{2}^{2}=1$. Then, Theorem 4 shows that the box spline $B\left(x \mid \frac{\Xi_{m, \ell}}{\sqrt{m-\ell / 2}}\right)$, and hence $\sqrt{m-\frac{\ell}{2}} B\left(\sqrt{m-\frac{\ell}{2}} x \mid \Xi_{m, \ell}\right)$, converges uniformly to the Gaussian function $\sqrt{\frac{6}{\pi}} \exp \left(-6 x^{2}\right)$.

Remark 3. A well-known result is that $\sqrt{m} B_{m}(\sqrt{m} x)$ converges uniformly to $\sqrt{\frac{6}{\pi}} \exp \left(-6 x^{2}\right)$ with $m \rightarrow \infty$ (see [11, 23]). In fact, the result can be considered as a particular case of Corollary 6. Note that

$$
\sqrt{m} \cdot B\left(\sqrt{m} x \mid \Xi_{m, 0}\right)=\sqrt{m} B_{m}(\sqrt{m} x) .
$$


If we take $\ell=0$ in Corollary 6 , then we have that $\sqrt{m} \cdot B\left(\sqrt{m} x \mid \Xi_{m, 0}\right)$, and hence $\sqrt{m} B_{m}(\sqrt{m} x)$, converges uniformly to $\sqrt{\frac{6}{\pi}} \exp \left(-6 x^{2}\right)$ with $m \rightarrow \infty$.

3.2. The asymptotic property of B-spline framelets. By changing variables, we can observe from Corollary 6 that $B\left(x \mid \Xi_{m, \ell}\right)$ is close to

$$
\sqrt{\frac{6}{\pi}} \frac{1}{\sqrt{m-\ell / 2}} \exp \left(-\frac{12 \cdot x^{2}}{2 m-\ell}\right) .
$$

Recall that Theorem 3 says that

$$
\psi_{\ell}^{(m)}(x)=\sqrt{\left(\begin{array}{c}
m \\
\ell
\end{array}\right)} \cdot \frac{1}{4^{\ell}} \cdot \frac{d^{\ell}}{d x^{\ell}} B\left(x-\left.\frac{j_{m}}{2}\right|_{m, \ell}\right) .
$$

Motivated by the two observations, we consider the relation between $\psi_{\ell}^{(m)}(x)$ and the $\ell$ th derivative of a Gaussian function $G(x)$, which is defined as

$$
G_{m, \ell}(x):=C_{m, \ell} \cdot \exp \left(-\frac{12 \cdot x^{2}}{2 m-\ell}\right)
$$

where

$$
C_{m, \ell}=\sqrt{\frac{6}{\pi}} \frac{\sqrt{\left(\begin{array}{c}
m \\
\ell
\end{array}\right)}}{\sqrt{m-\ell / 2} \cdot 4^{\ell}} .
$$

Let

$$
G_{\ell}^{(m)}(x):=\frac{d^{\ell}}{d x^{\ell}} G_{m, \ell}\left(x-\frac{j_{m}}{2}\right), \quad \ell=1, \ldots, m,
$$

where $j_{m}$ is given in (3), and

$$
G^{(m)}:=\left\{G_{1}^{(m)}, \ldots, G_{m}^{(m)}\right\} .
$$

Then we have

Theorem 7. Let $m \in \mathbb{N}$ be given and $1 \leq \ell \leq m$, and the framelet $\psi_{\ell}^{(m)}$ be defined by its Fourier transform in (4) derived from B-spline of order $m$. Then,

$$
\max _{1 \leq \ell \leq m} \max _{x \in \mathbb{R}}\left|\psi_{\ell}^{(m)}(x)-G_{\ell}^{(m)}(x)\right| \lesssim \frac{(\ln m)^{5 / 2}}{m^{3 / 2}} .
$$

In order to prove Theorem 7 we need the following two lemmas:

Lemma 8. The following inequality holds for every $\omega$, such that $|\omega| \geq$ $20 \sqrt{\frac{\ln m}{m}}$ :

$$
\max _{1 \leq \ell \leq m} \sqrt{\left(\begin{array}{c}
m \\
\ell
\end{array}\right)} \cdot \frac{1}{4^{\ell}} \cdot\left|\omega^{\ell} \cdot \exp \left(-\left(m-\frac{\ell}{2}\right) \frac{\omega^{2}}{24}\right)\right| \lesssim \frac{1}{m^{4}} .
$$


Proof. For the convenient, denote

$$
F_{\ell}(\omega):=\sqrt{\left(\begin{array}{c}
m \\
\ell
\end{array}\right)} \cdot \frac{1}{4^{\ell}} \cdot \omega^{\ell} \cdot \exp \left(-\left(m-\frac{\ell}{2}\right) \frac{\omega^{2}}{24}\right)
$$

and

$$
\omega_{\ell}:=\sqrt{\frac{24 \cdot \ell}{2 m-\ell}} .
$$

For each fixed $\ell \in[1, m] \cap \mathbb{Z}$, the function $F_{\ell}$ is increasing on the interval $\left[0, \omega_{\ell}\right]$, while $F_{\ell}$ is decreasing on $\left[\omega_{\ell}, \infty\right)$. And hence $F_{\ell}$ arrives at the maximum value at $\omega_{\ell}$. According to the inequality

$$
\left(\begin{array}{c}
m \\
\ell
\end{array}\right) \leq\left(\frac{m \cdot e}{\ell}\right)^{\ell}
$$

we have

$$
\ln F_{\ell}\left(\omega_{\ell}\right) \leq-\frac{\ell}{2} \cdot \ln \frac{2(2 m-\ell)}{3 m}
$$

Then, when

$$
\frac{2 \ln m}{\ln 16-\ln 15} \leq \ell \leq \frac{2}{5} m
$$

we have that

$$
\ln F_{\ell}\left(\omega_{\ell}\right) \leq-\frac{\ell}{2} \cdot \ln \frac{2(2 m-\ell)}{3 m} \leq-\frac{\ln \frac{4}{3}}{\ln \frac{16}{15}} \ln m \leq-4 \ln m .
$$

This implies that, whenever

$$
\frac{2 \ln m}{\ln 16-\ln 15} \leq \ell \leq \frac{2}{5} m
$$

holds, one has

$$
\max _{\omega} F_{\ell}(\omega) \leq F_{\ell}\left(\omega_{\ell}\right) \leq \frac{1}{m^{4}}
$$

We next consider the case where $\frac{2 m}{5} \leq \ell \leq \frac{4 m}{5}$. Using the inequality

$$
\left(\begin{array}{c}
m \\
\ell
\end{array}\right) \leq 2^{m}
$$

one gets that

$$
\ln F_{\ell}\left(\omega_{\ell}\right) \leq \frac{\ln 2}{2} m-\frac{\ell}{2} \ln \frac{2 e(2 m-\ell)}{3 \ell} .
$$

Therefore, when $\frac{2 m}{5} \leq \ell \leq \frac{4 m}{5}$, one has that

$$
\ln F_{\ell}\left(\omega_{\ell}\right) \leq \frac{\ln 2}{2} m-\frac{\ell}{2} \ln \frac{2 e(2 m-\ell)}{3 \ell} \lesssim-m \lesssim-4 \ln m,
$$

which implies that, whenever $\frac{2 m}{5} \leq \ell \leq \frac{4 m}{5}$, the following holds:

$$
\max _{\omega} F_{\ell}(\omega) \leq F_{\ell}\left(\omega_{\ell}\right) \lesssim \frac{1}{m^{4}} \text {. }
$$


We now turn to the case $\frac{4}{5} m \leq \ell \leq m$. For this case, we apply the inequality

$$
\left(\begin{array}{c}
m \\
\ell
\end{array}\right) \leq\left(\frac{m \cdot e}{m-\ell}\right)^{m-\ell}
$$

to obtain that

$$
\ln F_{\ell}\left(\omega_{\ell}\right) \leq \frac{m-\ell}{2} \ln \frac{m \cdot e}{m-\ell}-\frac{\ell}{2} \ln \frac{(4 m-2 \ell) \cdot e}{3 \ell} \lesssim-m \lesssim-4 \ln m .
$$

Hence, when $\frac{4}{5} m \leq \ell \leq m$, we have that

$$
\max _{\omega} F_{\ell}(\omega) \leq F_{\ell}\left(\omega_{\ell}\right) \lesssim \frac{1}{m^{4}}
$$

We finally consider the case where $1 \leq \ell \leq \frac{2 \ln m}{\ln 16-\ln 15}$. Note that, when $m$ is large enough, we have $\omega_{\ell} \leq 20 \sqrt{\frac{\ln m}{m}}$. When $|\omega| \geq 20 \sqrt{\frac{\ln m}{m}}, F_{\ell}(\omega)$ reach the maximum value at $\bar{\omega}_{0}:=20 \sqrt{\frac{\ln m}{m}}$. Then a simple calculation shows that, when $1 \leq \ell \leq \frac{2 \ln m}{\ln 16-\ln 15}$,

$$
\max _{|\omega| \geq 20 \sqrt{\frac{\ln m}{m}}} F_{\ell}(\omega) \leq F_{\ell}\left(\bar{\omega}_{0}\right) \lesssim \frac{1}{m^{4}}
$$

Combing (29), (30), (31) and (32), we conclude the proof.

Lemma 9. The following inequality holds for every $\omega \in \mathbb{R}$,

$$
\max _{1 \leq \ell \leq m} \frac{\sqrt{\left(\begin{array}{c}
m \\
\ell
\end{array}\right)}}{4^{\ell}} \cdot|\omega|^{\ell} \cdot\left|\operatorname{sinc}\left(\frac{\omega}{2}\right)^{m-\ell} \operatorname{sinc}\left(\frac{\omega}{4}\right)^{2 \ell}-\exp \left(-\frac{(m-\ell / 2) \omega^{2}}{24}\right)\right| \lesssim \frac{\ln ^{2} m}{m} \text {. }
$$

Proof. For the convenience, we only provide the proof for the case where $\omega \geq 0$. The proof of the other case is similar. By Taylor expansion, when $0 \leq \omega \leq \frac{3 \pi}{2}$, we have

$$
\begin{aligned}
& \operatorname{sinc}\left(\frac{\omega}{2}\right)^{m-\ell} \operatorname{sinc}\left(\frac{\omega}{4}\right)^{2 \ell} \\
= & \exp \left(-\frac{(m-\ell / 2) \omega^{2}}{24}\right) \exp \left(-\frac{(m-7 \ell / 8) \omega^{4}}{2880}-O\left(\omega^{6}\right)\right) .
\end{aligned}
$$

Then, for $20 \sqrt{\frac{\ln m}{m}} \leq \omega \leq \frac{3 \pi}{2}$, we have

$$
\begin{aligned}
& \frac{\sqrt{\left(\begin{array}{c}
m \\
\ell
\end{array}\right)}}{4^{\ell}} \cdot \omega^{\ell} \cdot\left|\operatorname{sinc}\left(\frac{\omega}{2}\right)^{m-\ell} \operatorname{sinc}\left(\frac{\omega}{4}\right)^{2 \ell}-\exp \left(-\frac{(m-\ell / 2) \omega^{2}}{24}\right)\right| \\
\lesssim & \frac{\sqrt{\left(\begin{array}{c}
m \\
\ell
\end{array}\right)}}{4^{\ell}} \cdot \omega^{\ell} \cdot \exp \left(-\frac{(m-\ell / 2) \omega^{2}}{24}\right)\left(1-\exp \left(-\frac{(m-7 \ell / 8) \omega^{4}}{2880}\right)\right) \\
\leq & 2 \frac{\sqrt{\left(\begin{array}{c}
m \\
\ell
\end{array}\right)}}{4^{\ell}} \cdot \omega^{\ell} \cdot \exp \left(-\frac{(m-\ell / 2) \omega^{2}}{24}\right) \lesssim \frac{1}{m^{4}},
\end{aligned}
$$


where the last inequality is obtained by Lemma 8 . Next, when $0 \leq \omega \leq$ $20 \sqrt{\frac{\ln m}{m}}$, note that

$$
1-\exp \left(-\frac{(m-7 \ell / 8) \omega^{4}}{2880}\right) \lesssim \frac{\ln ^{2} m}{m}
$$

and

$$
F_{\ell}(\omega)=\frac{\sqrt{\left(\begin{array}{c}
m \\
\ell
\end{array}\right)}}{4^{\ell}} \cdot \omega^{\ell} \cdot \exp \left(-\frac{(m-\ell / 2) \omega^{2}}{24}\right)
$$

is a bounded function. Hence, for $0 \leq \omega \leq 20 \sqrt{\frac{\ln m}{m}}$, we have

$$
\frac{\sqrt{\left(\begin{array}{c}
m \\
\ell
\end{array}\right)}}{4^{\ell}} \cdot \omega^{\ell} \cdot\left|\operatorname{sinc}\left(\frac{\omega}{2}\right)^{m-\ell} \operatorname{sinc}\left(\frac{\omega}{4}\right)^{2 \ell}-\exp \left(-\frac{(m-\ell / 2) \omega^{2}}{24}\right)\right| \lesssim \frac{\ln ^{2} m}{m} .
$$

Finally, We consider the case when $\omega \geq \frac{3 \pi}{2}$. We assert that, when $\omega \geq \frac{3 \pi}{2}$, the following inequality holds:

$$
\max _{1 \leq \ell \leq m} \sqrt{\left(\begin{array}{c}
m \\
\ell
\end{array}\right)} \cdot\left|\operatorname{sinc}\left(\frac{\omega}{2}\right)\right|^{m-\ell} \cdot\left|\operatorname{sinc}\left(\frac{\omega}{4}\right)\right|^{\ell} \leq\left(\frac{8 \cdot e^{1 / 8}}{3 \pi}\right)^{m} .
$$

With this assertion, we have

$$
\begin{aligned}
& \frac{\sqrt{\left(\begin{array}{c}
m \\
\ell
\end{array}\right)}}{4^{\ell}} \cdot \omega^{\ell} \cdot\left|\operatorname{sinc}\left(\frac{\omega}{2}\right)^{m-\ell} \operatorname{sinc}\left(\frac{\omega}{4}\right)^{2 \ell}-\exp \left(-\frac{(m-\ell / 2) \omega^{2}}{24}\right)\right| \\
\leq & \frac{\sqrt{\left(\begin{array}{c}
m \\
\ell
\end{array}\right)}}{4^{\ell}} \cdot \omega^{\ell} \cdot\left(\left|\operatorname{sinc}\left(\frac{\omega}{2}\right)^{m-\ell} \operatorname{sinc}\left(\frac{\omega}{4}\right)^{2 \ell}\right|+\left|\exp \left(-\frac{(m-\ell / 2) \omega^{2}}{24}\right)\right|\right) \\
\leq & \sqrt{\left(\begin{array}{c}
m \\
\ell
\end{array}\right)}\left(\left|\operatorname{sinc}\left(\frac{\omega}{2}\right)^{m-\ell} \operatorname{sinc}\left(\frac{\omega}{4}\right)^{\ell}\right|+\frac{1}{4^{\ell}} \cdot\left|\omega^{\ell} \cdot \exp \left(-\frac{(m-\ell / 2) \omega^{2}}{24}\right)\right|\right) \\
\lesssim & \frac{1}{m^{4}} .
\end{aligned}
$$

Here, the last inequality is followed by (33) and Lemma 8 . It remains to prove (33). Note that, when $\omega \geq \frac{3 \pi}{2}$,

$$
\left|\operatorname{sinc}\left(\frac{\omega}{2}\right)\right|^{m-\ell} \cdot\left|\operatorname{sinc}\left(\frac{\omega}{4}\right)\right|^{\ell} \leq \frac{1}{(\omega / 2)^{m-\ell}} \cdot \frac{1}{(\omega / 4)^{\ell}} \leq \frac{1}{(3 \pi / 4)^{m-\ell}} \cdot \frac{1}{(3 \pi / 8)^{\ell}} .
$$

Then we only need prove

$$
\max _{1 \leq \ell \leq m} \sqrt{\left(\begin{array}{c}
m \\
\ell
\end{array}\right)} \cdot \frac{1}{(3 \pi / 4)^{m-\ell}} \cdot \frac{1}{(3 \pi / 8)^{\ell}} \lesssim\left(\frac{8 \cdot e^{1 / 8}}{3 \pi}\right)^{m} .
$$


Applying the inequality $\left(\begin{array}{c}m \\ \ell\end{array}\right) \leq\left(\frac{m \cdot e}{m-\ell}\right)^{m-\ell}$, we have that,

$$
\max _{1 \leq \ell \leq m} \sqrt{\left(\begin{array}{c}
m \\
\ell
\end{array}\right)} \cdot \frac{1}{(3 \pi / 4)^{m-\ell}} \cdot \frac{1}{(3 \pi / 8)^{\ell}} \leq\left(\frac{8 \cdot e^{1 / 8}}{3 \pi}\right)^{m} .
$$

This proves that (33).

Proof of Theorem [7, Let

$M(\omega):=\max _{1 \leq \ell \leq m} \frac{\sqrt{\left(\begin{array}{c}m \\ \ell\end{array}\right)}}{4^{\ell}} \cdot|\omega|^{\ell} \cdot\left|\operatorname{sinc}\left(\frac{\omega}{2}\right)^{m-\ell} \operatorname{sinc}\left(\frac{\omega}{4}\right)^{2 \ell}-\exp \left(-\left(m-\frac{\ell}{2}\right) \frac{\omega^{2}}{24}\right)\right|$,

and

$$
\begin{aligned}
& I_{1}:=\left\{\omega \in \mathbb{R}:|\omega| \leq 20 \sqrt{\frac{\ln m}{m}}\right\}, I_{2}:=\left\{\omega \in \mathbb{R}: 20 \sqrt{\frac{\ln m}{m}} \leq|\omega| \leq \frac{3 \pi}{2}\right\}, \\
& I_{3}:=\left\{\omega \in \mathbb{R}:|\omega| \geq \frac{3 \pi}{2}\right\} .
\end{aligned}
$$

Applying Lemma 9 and Lemma 8, we conclude that

$$
\int_{I_{1}} M(\omega) d \omega \lesssim \frac{(\ln m)^{5 / 2}}{m^{3 / 2}}, \quad \int_{I_{2}} M(\omega) d \omega \lesssim \frac{1}{m^{4}}
$$

respectively. By an argument similar to that leading to (33), we can obtain that there exists $0<\gamma<1$ such that

$$
\int_{I_{3}} M(\omega) d \omega \lesssim \gamma^{m} \lesssim \frac{(\ln m)^{5 / 2}}{m^{3 / 2}}
$$

This leads to that

$$
\begin{aligned}
& \max _{1 \leq \ell \leq m} \max _{x \in \mathbb{R}}\left|\psi_{\ell}^{(m)}(x)-G_{\ell}^{(m)}(x)\right| \leq \int_{-\infty}^{\infty} M(\omega) d \omega \\
= & \int_{I_{1}} M(\omega) d \omega+\int_{I_{2}} M(\omega) d \omega+\int_{I_{3}} M(\omega) d \omega \lesssim \frac{(\ln m)^{5 / 2}}{m^{3 / 2}} .
\end{aligned}
$$

Remark 4. It was proved in [17] that, for each fixed $\ell$, up to a normalization, a proper scaled $\psi_{\ell}^{(m)}$ uniformly converges to the $\ell$-order derivative of a scaled Gaussian function with $m$ tending to infinity. Our result is in a different direction. In fact, we show that for sufficiently large $m$ framelets $\psi_{1}^{(m)}, \ldots, \psi_{\ell}^{(m)}, \ldots, \psi_{m}^{(m)}$ uniformly in $x$ and $\ell$ close to derivatives of consecutive orders $1, \ldots, m$ of a scaled Gaussian function whose scale depends on $m$. 


\section{Gaussian frame}

Theorem 7 leads us to consider whether a wavelet system generated by a finite number of consecutive derivatives of a properly scaled Gaussian function forms a frame of $L_{2}(\mathbb{R})$. In this section, we show that the frame property of $X\left(\psi_{1}^{(m)}, \ldots, \psi_{m}^{(m)}\right)$ can be transferred to that of $X\left(G_{1}^{(m)}, \ldots, G_{m}^{(m)}\right)$ where $G_{\ell}^{(m)}$ is defined as follows: For each fixed $m \in \mathbb{N}$, we consider the following rescaled Gaussian function

$$
G_{m, \ell}(x)=C_{m, \ell} \cdot \exp \left(-\frac{12 \cdot x^{2}}{2 m-\ell}\right)
$$

where

$$
C_{m, \ell}=\sqrt{\frac{6}{\pi}} \frac{\sqrt{\left(\begin{array}{c}
m \\
\ell
\end{array}\right)}}{\sqrt{m-\ell / 2} \cdot 4^{\ell}} ;
$$

and

$$
G_{\ell}^{(m)}(x)=\frac{d^{\ell}}{d x^{\ell}} G_{m, \ell}\left(x-\frac{j_{m}}{2}\right), \quad \ell=1, \ldots, m,
$$

where $j_{m}$ is given in (3), and

$$
G^{(m)}=\left\{G_{1}^{(m)}, \ldots, G_{m}^{(m)}\right\} .
$$

Before stating the following main theorem of this section, we recall the definitions of the frame and Bessel sequence. A family $\left\{f_{j}\right\}_{j \in J} \subset L_{2}(\mathbb{R})$ is called a frame with bounds $A$ and $B$ if

$$
A\|f\|^{2} \leq \sum_{j \in J}\left|\left\langle f, f_{j}\right\rangle\right|^{2} \leq B\|f\|^{2}
$$

holds for all $f \in L_{2}(\mathbb{R})$. If $A=B$, then $\left\{f_{j}\right\}_{j \in J}$ is called a $A$-tight frame. Moreover, a family $\left\{f_{j}\right\}_{j \in J} \subset L_{2}(\mathbb{R})$ is called a Bessel sequence with a bound $R$ if

$$
\sum_{j \in J}\left|\left\langle f, f_{j}\right\rangle\right|^{2} \leq R\|f\|^{2}
$$

holds for all $f \in L_{2}(\mathbb{R})$.

Theorem 10. Let $X\left(G^{(m)}\right)$ be the wavelet system generated by functions $G^{(m)}$. Then $X\left(G^{(m)}\right)$ is a frame system with frame bounds $A_{m}$ and $B_{m}$ for sufficiently large $m$. Furthermore, the frame is close to be tight as $m$ is sufficiently large. In fact, asymptotically, we have

$$
\lim _{m \rightarrow \infty} A_{m}=\lim _{m \rightarrow \infty} B_{m}=1 .
$$

To prove Theorem 10, we need the following theorem, which is proven in [16], together with several lemmas.

Theorem 11 ( [16]). Let $\left\{f_{j}\right\}_{j \in J}$ be a frame of $L_{2}(\mathbb{R})$ with bounds $A$ and $B$. Assume that $\left\{g_{j}\right\}_{j \in J} \subset L_{2}(\mathbb{R})$ is such that $\left\{f_{j}-g_{j}\right\}_{j \in J}$ is a Bessel sequence 
with a bound $R<A$. Then $\left\{g_{j}\right\}_{j \in J}$ is a frame with bounds $A\left(1-\sqrt{\frac{R}{A}}\right)^{2}$ and $B\left(1+\sqrt{\frac{R}{B}}\right)^{2}$.

Let

$$
\phi_{\ell}^{(m)}:=\psi_{\ell}^{(m)}-G_{\ell}^{(m)}, \ell=1, \ldots, m, \quad \text { and } \quad \Phi^{(m)}:=\left\{\phi_{1}^{(m)}, \ldots, \phi_{m}^{(m)}\right\} .
$$

Since $X\left(\Psi^{(m)}\right)$ is a tight frame with frame bound 1, to prove that $X\left(G^{(m)}\right)$ is a frame, according to Theorem [1], we only need to show that $X\left(\Phi^{(m)}\right)$ is a Bessel sequence with a bound $R_{m} \rightarrow 0$. An estimate of the Bessel bound of a given a sequence is provided in [21] that enables us to estimate the Bessel bound of $X\left(\Phi^{(m)}\right)$ (see also [12]). Let

$$
R_{m}:=\sup _{1 \leq|\omega| \leq 2} \sum_{k \in \mathbb{Z}} \sum_{n \in \mathbb{Z}} \sum_{\ell=1}^{m}\left|\hat{\phi}_{\ell}^{(m)}\left(2^{n} \omega\right)\right| \cdot\left|\hat{\phi}_{\ell}^{(m)}\left(2^{n} \omega+2 k \pi\right)\right| .
$$

Then, for arbitrary $f \in L^{2}(\mathbb{R})$, the following inequality holds

$$
\sum_{\phi \in X\left(\Phi^{(m)}\right)}|\langle f, \phi\rangle|^{2} \leq R_{m}\|f\|_{2}^{2},
$$

i.e. $R_{m}$ is the Bessel up bound of the system $X\left(\Phi^{(m)}\right)$. Next, we estimate $R_{m}$. For this, we need the following lemmas.

Lemma 12. Let $\hat{\phi}_{\ell}^{(m)}$ be the Fourier transform of $\phi_{\ell}^{(m)}$ defined in (34). Then the following three estimates for $\left|\hat{\phi}_{\ell}^{(m)}\right|$ holds:

(i)

(ii)

$$
\left|\hat{\phi}_{\ell}^{(m)}(\omega)\right| \leq \sqrt{\left(\begin{array}{c}
m \\
\ell
\end{array}\right)} \cdot \frac{2^{m+\ell+1}}{|\omega|^{m}}, \quad|\omega| \geq 20 .
$$

$$
\left|\hat{\phi}_{\ell}^{(m)}(\omega)\right| \lesssim \sqrt{\left(\begin{array}{c}
m \\
\ell
\end{array}\right)}\left(\frac{\omega}{4}\right)^{\ell} \cdot m \cdot \omega^{4}, \quad|\omega| \leq \sqrt{\frac{1}{m}} .
$$

(iii)

$$
\max _{1 \leq \ell \leq m} \max _{\omega \in \mathbb{R}}\left|\hat{\phi}_{\ell}^{(m)}(\omega)\right| \lesssim \frac{\ln ^{2} m}{m} .
$$

Proof. First a simple calculation leads to

$$
\begin{aligned}
\left|\hat{\phi}_{\ell}^{(m)}(\omega)\right| & =\left|\hat{\psi}_{\ell}^{(m)}(\omega)-\hat{G}_{\ell}^{(m)}(\omega)\right| \\
& =\left|\sqrt{\left(\begin{array}{c}
m \\
\ell
\end{array}\right)} \frac{\omega^{\ell}}{4^{\ell}}\left(\operatorname{sinc}\left(\frac{\omega}{2}\right)^{m-\ell} \operatorname{sinc}\left(\frac{\omega}{4}\right)^{2 \ell}-\exp \left(-\left(m-\frac{\ell}{2}\right) \frac{\omega^{2}}{24}\right)\right)\right| .
\end{aligned}
$$


For (i), when $|\omega| \geq 20$, a simple argument shows that

$\left|\frac{\omega^{\ell}}{4^{\ell}}\right| \exp \left(-\left(m-\frac{\ell}{2}\right) \frac{\omega^{2}}{24}\right) \leq \frac{2^{m+\ell}}{|\omega|^{m}}, \quad\left|\left(\frac{\omega}{4}\right)^{\ell} \cdot \operatorname{sinc}\left(\frac{\omega}{2}\right)^{m-\ell} \cdot \operatorname{sinc}\left(\frac{\omega}{4}\right)^{2 \ell}\right| \leq \frac{2^{m+\ell}}{|\omega|^{m}}$,

which implies that

$$
\left|\hat{\phi}_{\ell}^{(m)}(\omega)\right| \leq \sqrt{\left(\begin{array}{c}
m \\
\ell
\end{array}\right)} \cdot \frac{2^{m+\ell+1}}{|\omega|^{m}} .
$$

For (ii), the Taylor expansion shows that, when $|\omega| \leq \pi$,

$$
\ln \left(\operatorname{sinc}\left(\frac{\omega}{2}\right)^{m-\ell} \operatorname{sinc}\left(\frac{\omega}{4}\right)^{2 \ell}\right)=-\left(\left(m-\frac{\ell}{2}\right) \frac{\omega^{2}}{24}+\left(m-\frac{7}{8} \ell\right) \frac{\omega^{4}}{2880}+O\left(\omega^{6}\right)\right)
$$

Then, when $|\omega| \leq \sqrt{\frac{1}{m}}$,

$$
\begin{aligned}
& \left|\operatorname{sinc}\left(\frac{\omega}{2}\right)^{m-\ell} \operatorname{sinc}\left(\frac{\omega}{4}\right)^{2 \ell}-\exp \left(-\left(m-\frac{\ell}{2}\right) \frac{\omega^{2}}{24}\right)\right| \\
= & \exp \left(-\left(m-\frac{\ell}{2}\right) \frac{\omega^{2}}{24}\right)\left|\exp \left(-\left(m-\frac{7}{8} \ell\right) \frac{\omega^{4}}{2880}-O\left(\omega^{6}\right)\right)-1\right| \\
\lesssim & m \cdot \omega^{4},
\end{aligned}
$$

which implies that

$$
\left|\hat{\phi}_{\ell}^{(m)}(\omega)\right| \lesssim \sqrt{\left(\begin{array}{c}
m \\
\ell
\end{array}\right)} \cdot\left(\frac{\omega}{4}\right)^{\ell} \cdot m \cdot \omega^{4} .
$$

Finally, the conclusion of (iii) can be obtained by Lemma 9 directly.

Lemma 13. Let $R_{m}$ be given by (35). Then

$$
R_{m} \lesssim \frac{\ln ^{5} m}{m} ; \text { and } \quad \lim _{m \rightarrow \infty} R_{m}=0 .
$$

Proof. Let

$$
\begin{aligned}
R_{m, 1} & :=\sup _{1 \leq|\omega| \leq 2} \sum_{n \in \mathbb{Z}} \sum_{\ell=1}^{m}\left|\hat{\phi}_{\ell}^{(m)}\left(2^{n} \omega\right)\right|^{2}, \\
R_{m, 2} & :=\sup _{1 \leq|\omega| \leq 2} \sum_{k \in \mathbb{Z} \backslash\{0\}} \sum_{n \in \mathbb{Z}} \sum_{\ell=1}^{m}\left(\left|\hat{\phi}_{\ell}^{(m)}\left(2^{n} \omega\right)\right| \cdot\left|\hat{\phi}_{\ell}^{(m)}\left(2^{n} \omega+2 k \pi\right)\right|\right) .
\end{aligned}
$$

Then, we have that

$$
R_{m} \leq R_{m, 1}+R_{m, 2} .
$$

To estimate $R_{m}$, we consider $R_{m, 1}$ and $R_{m, 2}$, respectively. We first estimate $R_{m, 1}$. For this, we rewrite

$$
R_{m, 1}=\sup _{1 \leq|\omega| \leq 2} \sum_{n \in \mathbb{Z}} \sum_{\ell=1}^{m}\left|\hat{\phi}_{\ell}^{(m)}\left(2^{n} \omega\right)\right|^{2}=\sup _{1 \leq|\omega| \leq 2}\left[S_{1}(\omega)+S_{2}(\omega)+S_{3}(\omega)\right]
$$


where

$$
\begin{aligned}
S_{1}(\omega) & :=\sum_{n \geq 5} \sum_{\ell=1}^{m}\left|\hat{\phi}_{\ell}^{(m)}\left(2^{n} \omega\right)\right|^{2}, \quad S_{2}(\omega):=\sum_{-\left\lfloor\log _{2} m\right\rfloor<n<5} \sum_{\ell=1}^{m}\left|\hat{\phi}_{\ell}^{(m)}\left(2^{n} \omega\right)\right|^{2}, \\
S_{3}(\omega) & :=\sum_{n \leq-\left\lfloor\log _{2} m\right\rfloor} \sum_{\ell=1}^{m}\left|\hat{\phi}_{\ell}^{(m)}\left(2^{n} \omega\right)\right|^{2} .
\end{aligned}
$$

By (i) in Lemma 12, we obtain that, for $1 \leq|\omega| \leq 2$,

$$
\begin{aligned}
S_{1}(\omega) & =\sum_{n \geq 5} \sum_{\ell=1}^{m}\left|\hat{\phi}_{\ell}^{(m)}\left(2^{n} \omega\right)\right|^{2} \\
& \leq 4 \sum_{n \geq 5} \sum_{\ell=1}^{m}\left(\begin{array}{c}
m \\
\ell
\end{array}\right) \frac{4^{m+\ell}}{\left(2^{n} \omega\right)^{2 m}} \\
& =4 \sum_{n \geq 5} \frac{4^{m}}{\left(2^{n} \omega\right)^{2 m}} \sum_{\ell=1}^{m}\left(\begin{array}{c}
m \\
\ell
\end{array}\right) 4^{\ell} \\
& \lesssim\left(\frac{5}{256}\right)^{m} .
\end{aligned}
$$

Using (ii) in Lemma 12, when $1 \leq|\omega| \leq 2$,

$$
\begin{aligned}
S_{3}(\omega) & =\sum_{n \leq-\left\lfloor\log _{2} m\right\rfloor} \sum_{\ell=1}^{m}\left|\hat{\phi}_{\ell}^{(m)}\left(2^{n} \omega\right)\right|^{2}=\sum_{n \geq\left\lfloor\log _{2} m\right\rfloor} \sum_{\ell=1}^{m}\left|\hat{\phi}_{\ell}^{(m)}\left(\frac{\omega}{2^{n}}\right)\right|^{2} \\
& \lesssim \sum_{n \geq\left\lfloor\log _{2} m\right\rfloor} \sum_{\ell=1}^{m}\left(\begin{array}{c}
m \\
\ell
\end{array}\right)\left(\frac{\omega}{4 \cdot 2^{n}}\right)^{2 \ell} \cdot m^{2} \cdot\left(\frac{\omega^{4}}{2^{4 n}}\right)^{2} \\
& =\sum_{n \geq\left\lfloor\log _{2} m\right\rfloor} m^{2} \cdot\left(\frac{\omega^{4}}{2^{4 n}}\right)^{2} \sum_{\ell=1}^{m}\left(\begin{array}{c}
m \\
\ell
\end{array}\right)\left(\frac{\omega}{4 \cdot 2^{n}}\right)^{2 \ell} \\
& \leq m^{2} \sum_{n \geq\left\lfloor\log _{2} m\right\rfloor}\left(\frac{\omega^{4}}{2^{4 n}}\right)^{2}\left(1+\left(\frac{\omega}{4 \cdot 2^{n}}\right)^{2}\right)^{m} \\
& \lesssim m^{2} \sum_{n \geq\left\lfloor\log _{2} m\right\rfloor}\left(\frac{1}{2^{4 n}}\right)^{2}\left(1+\left(\frac{1}{2^{n+1}}\right)^{2}\right)^{m} \\
& \lesssim \frac{1}{m^{6}} .
\end{aligned}
$$

Here, the last inequality uses the fact of $\left\{\left(1+\left(\frac{1}{2^{n+1}}\right)^{2}\right)^{m}\right\}_{n \geq\left\lfloor\log _{2} m\right\rfloor, m \in \mathbb{Z}_{+}}$ being a bounded sequence and

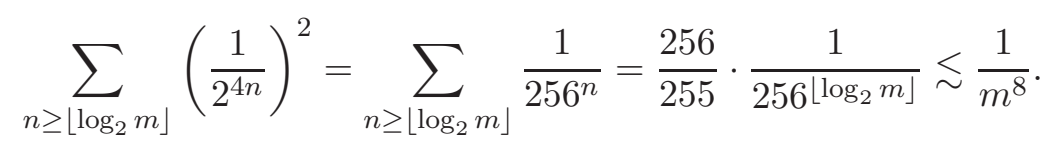


Moreover, by (iii) in Lemma 12, we have

$$
S_{3}(\omega)=\sum_{\ell=1}^{m} \sum_{-\left\lfloor\log _{2} m\right\rfloor \leq n \leq 5}\left|\hat{\phi}_{\ell}\left(2^{n} \omega\right)\right|^{2} \lesssim \frac{\ln ^{5} m}{m} .
$$

Combining the results above, we obtain that

$$
R_{m, 1}=\sup _{1 \leq|\omega| \leq 2}\left[S_{1}(\omega)+S_{2}(\omega)+S_{3}(\omega)\right] \lesssim \frac{\ln ^{5} m}{m} .
$$

We next turn to

$$
R_{m, 2}=\sup _{1 \leq|\omega| \leq 2} \sum_{k \in \mathbb{Z} \backslash\{0\}} \sum_{\ell=1}^{m} \sum_{n \in \mathbb{Z}}\left(\left|\hat{\phi}_{\ell}^{(m)}\left(2^{n} \omega\right)\right| \cdot\left|\hat{\phi}_{\ell}^{(m)}\left(2^{n} \omega+2 k \pi\right)\right|\right) .
$$

To state conveniently, we set

$$
\beta(2 k \pi):=\sup _{1 \leq|\omega| \leq 2} \sum_{\ell=1}^{m} \sum_{n \in \mathbb{Z}}\left|\hat{\phi}_{\ell}^{(m)}\left(2^{n} \omega\right)\right| \cdot\left|\hat{\phi}_{\ell}^{(m)}\left(2^{n} \omega+2 k \pi\right)\right| .
$$

Then

$$
R_{m, 2} \leq \sum_{k \in \mathbb{Z} \backslash\{0\}} \beta(2 k \pi) .
$$

Set $k_{0}:=10$. When $1 \leq|k| \leq k_{0}-1$, using the argument similar to the one in the estimation of $R_{m, 1}=\beta(0)$, we can show that

$$
\beta(2 k \pi) \lesssim \frac{1}{m} \quad \text { for } 1 \leq|k| \leq k_{0}-1
$$

We claim that, when $|k| \geq k_{0}$,

$$
\beta(2 k \pi) \lesssim \frac{3^{m}}{|2 k \pi|^{m / 2}}
$$

And hence,

$$
R_{m, 2}=\left(\sum_{1 \leq|k| \leq k_{0}-1}+\sum_{|k| \geq k_{0}}\right) \beta(2 k \pi) \lesssim \frac{1}{m}+\sum_{|k| \geq k_{0}} \frac{3^{m}}{|2 k \pi|^{m / 2}} \lesssim \frac{1}{m}
$$

Combing (37) and (39), we obtain that

$$
R_{m}=R_{m, 1}+R_{m, 2} \lesssim \frac{\ln ^{5} m}{m},
$$

which implies the conclusion.

Finally, we prove (38). A simple observation is that $\beta(2 k \pi)=\beta(-2 k \pi)$. Hence, we only need consider the case where $k \geq k_{0}$. For the convenience, 
let

$$
\begin{aligned}
& \beta_{+}(2 k \pi):=\sup _{1 \leq|\omega| \leq 2} \sum_{\ell=1}^{m} \sum_{n \in \mathbb{Z}_{+}}\left(\left|\hat{\phi}_{\ell}^{(m)}\left(2^{n} \omega\right)\right| \cdot\left|\hat{\phi}_{\ell}^{(m)}\left(2^{n} \omega+2 k \pi\right)\right|\right), \\
& \beta_{-}(2 k \pi):=\sup _{1 \leq|\omega| \leq 2} \sum_{\ell=1}^{m} \sum_{n<0}\left(\left|\hat{\phi}_{\ell}^{(m)}\left(2^{n} \omega\right)\right| \cdot\left|\hat{\phi}_{\ell}^{(m)}\left(2^{n} \omega+2 k \pi\right)\right|\right) .
\end{aligned}
$$

Then $\beta(2 k \pi) \leq \beta_{+}(2 k \pi)+\beta_{-}(2 k \pi)$. To estimate $\beta_{+}(2 k \pi)$, we furthermore set

$$
\begin{aligned}
& \beta_{+}^{+}(2 k \pi):=\sup _{1 \leq \omega \leq 2} \sum_{\ell=1}^{m} \sum_{n \in \mathbb{Z}_{+}}\left|\hat{\phi}_{\ell}^{(m)}\left(2^{n} \omega\right)\right| \cdot\left|\hat{\phi}_{\ell}^{(m)}\left(2^{n} \omega+2 k \pi\right)\right|, \\
& \beta_{+}^{-}(2 k \pi):=\sup _{-2 \leq \omega \leq-1} \sum_{\ell=1}^{m} \sum_{n \in \mathbb{Z}_{+}}\left|\hat{\phi}_{\ell}^{(m)}\left(2^{n} \omega\right)\right| \cdot\left|\hat{\phi}_{\ell}^{(m)}\left(2^{n} \omega+2 k \pi\right)\right| .
\end{aligned}
$$

Then $\beta_{+}(2 k \pi)=\max \left\{\beta_{+}^{+}(2 k \pi), \beta_{+}^{-}(2 k \pi)\right\}$. Note that $k \geq k_{0}=10$, by Lemma 12,

$$
\begin{aligned}
\beta_{+}^{+}(2 k \pi) & =\sup _{1 \leq \omega \leq 2} \sum_{\ell=1}^{m} \sum_{n \in \mathbb{Z}_{+}}\left|\hat{\phi}_{\ell}^{(m)}\left(2^{n} \omega\right)\right| \cdot\left|\hat{\phi}_{\ell}^{(m)}\left(2^{n} \omega+2 k \pi\right)\right| \\
& \lesssim \sum_{\ell=1}^{m} \sum_{n \in \mathbb{Z}_{+}}\left(\begin{array}{c}
m \\
\ell
\end{array}\right) \frac{2^{m+\ell}}{\left(2^{n}+2 k \pi\right)^{m}} \\
& =\sum_{n \in \mathbb{Z}_{+}} \sum_{\ell=1}^{m}\left(\begin{array}{c}
m \\
\ell
\end{array}\right) \frac{2^{m+\ell}}{\left(2^{n}+2 k \pi\right)^{m}} \\
& \leq \sum_{n \in \mathbb{Z}_{+}} \frac{6^{m}}{\left(2^{n}+2 k \pi\right)^{m}} \leq \sum_{n \in \mathbb{Z}_{+}} \frac{6^{m}}{2^{m} \cdot 2^{n m / 2} \cdot(2 k \pi)^{m / 2}} \\
& \lesssim \frac{3^{m}}{(2 k \pi)^{m / 2}}
\end{aligned}
$$

We next consider

$$
\begin{aligned}
\beta_{+}^{-}(2 k \pi) & =\sup _{-2 \leq \omega \leq-1} \sum_{\ell=1}^{m} \sum_{n \in \mathbb{Z}_{+}}\left|\hat{\phi}_{\ell}^{(m)}\left(2^{n} \omega\right)\right| \cdot\left|\hat{\phi}_{\ell}^{(m)}\left(2^{n} \omega+2 k \pi\right)\right| \\
& =\sup _{1 \leq \omega \leq 2} \sum_{\ell=1}^{m} \sum_{n \in \mathbb{Z}_{+}}\left|\hat{\phi}_{\ell}^{(m)}\left(2^{n} \omega\right)\right| \cdot\left|\hat{\phi}_{\ell}^{(m)}\left(2^{n} \omega-2 k \pi\right)\right| .
\end{aligned}
$$

A simple observation is that $\max \left\{\left|2^{n} \omega\right|,\left|2^{n} \omega-2 k \pi\right|\right\} \geq k \pi \geq k_{0} \pi$. Then using (i) in Lemma 12, we obtain that

$$
\left|\hat{\phi}_{\ell}^{(m)}\left(2^{n} \omega\right)\right| \cdot\left|\hat{\phi}_{\ell}^{(m)}\left(2^{n} \omega-2 k \pi\right)\right| \lesssim\left(\begin{array}{c}
m \\
\ell
\end{array}\right) \cdot \frac{2^{m+\ell}}{(k \pi)^{m}} .
$$


Set $n_{0}:=\left\lceil\log _{2}(2 k \pi)\right\rceil+5$. Then,

$$
\begin{aligned}
& \sup _{1 \leq \omega \leq 2} \sum_{\ell=1}^{m} \sum_{0 \leq n \leq n_{0}}\left|\hat{\phi}_{\ell}^{(m)}\left(2^{n} \omega\right)\right| \cdot\left|\hat{\phi}_{\ell}^{(m)}\left(2^{n} \omega-2 k \pi\right)\right| \\
\lesssim & \sum_{0 \leq n \leq n_{0}} \sum_{\ell=1}^{m}\left(\begin{array}{c}
m \\
\ell
\end{array}\right) \frac{2^{m+\ell}}{(k \pi)^{m}}=\sum_{0 \leq n \leq n_{0}} \frac{2^{m}}{(k \pi)^{m}} \sum_{\ell=1}^{m}\left(\begin{array}{c}
m \\
\ell
\end{array}\right) \cdot 2^{\ell} \\
\leq & \sum_{0 \leq n \leq n_{0}} \frac{6^{m}}{(k \pi)^{m}} \lesssim \log _{2}(2 k \pi) \cdot \frac{6^{m}}{(k \pi)^{m}} .
\end{aligned}
$$

We next consider

$$
\sup _{1 \leq \omega \leq 2} \sum_{\ell=1}^{m} \sum_{n_{0}+1 \leq n}\left|\hat{\phi}_{\ell}^{(m)}\left(2^{n} \omega\right)\right| \cdot\left|\hat{\phi}_{\ell}^{(m)}\left(2^{n} \omega-2 k \pi\right)\right| .
$$

By (i) in Lemma 12, when $n \geq n_{0}+1$,

$$
\sup _{1 \leq \omega \leq 2}\left|\hat{\phi}_{\ell}^{(m)}\left(2^{n} \omega\right)\right| \leq \sqrt{\left(\begin{array}{c}
m \\
\ell
\end{array}\right)} \frac{2^{m+\ell}}{(2 k \pi)^{m}}, \sup _{1 \leq \omega \leq 2}\left|\hat{\phi}_{\ell}^{(m)}\left(2^{n} \omega-2 k \pi\right)\right| \leq \sqrt{\left(\begin{array}{c}
m \\
\ell
\end{array}\right)} \frac{2^{m+\ell}}{\left(2^{n}-2 k \pi\right)^{m}} .
$$

Hence,

$$
\begin{aligned}
& \sup _{1 \leq \omega \leq 2} \sum_{\ell=1}^{m} \sum_{n \geq n_{0}+1}\left|\hat{\phi}_{\ell}^{(m)}\left(2^{n} \omega\right)\right| \cdot\left|\hat{\phi}_{\ell}^{(m)}\left(2^{n} \omega-2 k \pi\right)\right| \\
\leq & \sum_{\ell=1}^{m} \sum_{n \geq n_{0}+1}\left(\begin{array}{c}
m \\
\ell
\end{array}\right) \frac{2^{m+\ell}}{(2 k \pi)^{m}} \cdot \frac{2^{m+\ell}}{\left(2^{n}-2 k \pi\right)^{m}} \\
\leq & \frac{4^{m} \cdot 5^{m}}{(2 k \pi)^{m}} \sum_{n \geq n_{0}+1} \frac{1}{\left(2^{n}-2 k \pi\right)^{m}} \lesssim\left(\frac{10}{k \pi}\right)^{m}, \quad k \geq k_{0} .
\end{aligned}
$$

Therefore,

$$
\beta_{+}^{-}(2 k \pi) \lesssim \log _{2}(2 k \pi) \cdot \frac{6^{m}}{(k \pi)^{m}}+\left(\frac{10}{k \pi}\right)^{m} .
$$

This concludes that

$$
\beta_{+}(2 k \pi)=\max \left\{\beta_{+}^{+}(2 k \pi), \beta_{+}^{-}(2 k \pi)\right\} \lesssim \frac{3^{m}}{(2 k \pi)^{m / 2}} .
$$

Using (ii) in Lemma 12 and a similar analysis with above, we can obtain that

$$
\begin{aligned}
\beta_{-}(2 k \pi) & =\sup _{1 \leq|\omega| \leq 2} \sum_{\ell=1}^{m} \sum_{n<0}\left|\hat{\phi}_{\ell}^{(m)}\left(2^{n} \omega\right)\right| \cdot\left|\hat{\phi}_{\ell}^{(m)}\left(2^{n} \omega+2 k \pi\right)\right| \\
& \lesssim m \cdot \frac{4^{m}}{(2 k \pi)^{m}}
\end{aligned}
$$


TABLE 1. The numerical results of frame bounds of $X\left(G^{(m)}\right)$

\begin{tabular}{cccccccc}
\hline$m$ & 2 & 3 & 4 & 5 & 6 & 7 & 8 \\
\hline$A$ & 0.3855 & 0.5266 & 0.5898 & 0.6407 & 0.6803 & 0.7095 & 0.7274 \\
$B$ & 1.9020 & 1.6239 & 1.5179 & 1.4390 & 1.3811 & 1.3403 & 1.3159 \\
\hline
\end{tabular}

Putting everything together, we have that

$$
\beta(2 k \pi)=\beta_{+}(2 k \pi)+\beta_{-}(2 k \pi) \lesssim \frac{6^{m}}{(2 \sqrt{2 k \pi})^{m}}+m \cdot \frac{4^{m}}{(2 k \pi)^{m}} \lesssim \frac{3^{m}}{(2 k \pi)^{m / 2}} .
$$

This proves (38).

Proof of Theorem 10, Recall that

$$
\Phi^{(m)}=\left\{\phi_{1}^{(m)}, \ldots, \phi_{m}^{(m)}\right\}, \phi_{\ell}^{(m)}=\psi_{\ell}^{(m)}-G_{\ell}^{(m)}, \quad \ell=1, \ldots, m ;
$$

and that $X\left(\Psi^{(m)}\right)$ is a tight frame with frame bound 1 , where $\Psi^{(m)}=$ $\left\{\psi_{1}^{(m)}, \ldots, \psi_{m}^{(m)}\right\}$. Lemma 13 shows that $X\left(\Phi^{(m)}\right)$ is a Bessel sequence with a bound $R_{m} \rightarrow 0$. Then Theorem 11 leads that $X\left(G^{(m)}\right)$ is a frame with frame bound $A_{m}=\left(1-\sqrt{R_{m}}\right)^{2}$ and $B_{m}=\left(1+\sqrt{R_{m}}\right)^{2}$ as $m$ sufficiently large. Furthermore, it can be close to a tight frame, since $\lim _{m \rightarrow \infty} A_{m}=$ $\lim _{m \rightarrow \infty} B_{m}=1$, which completes the proof.

Remark 5. Although Theorem 10 confirms the case where $m$ is sufficiently large, the result of Theorem 10 seems to hold for small $m$ ( $m$ can be as small as 2). For small $m$, combining (35) and Theorem 11, we can estimate the frame bounds of $X\left(G^{(m)}\right)$ numerically. We list the frame bound estimation of $X\left(G^{(m)}\right), 2 \leq m \leq 8$, in Table 1 , which clearly shows the frame property of $X\left(G^{(m)}\right)$ for small $m$. For example, for $m=2, X\left(G^{(2)}\right)$ is a frame with frame bounds $A \approx 0.3855$ and $B \approx 1.9020$. Figure 2 shows that the graphs of the functions of $G_{1}^{(2)}$ and $G_{2}^{(2)}$, respectively.
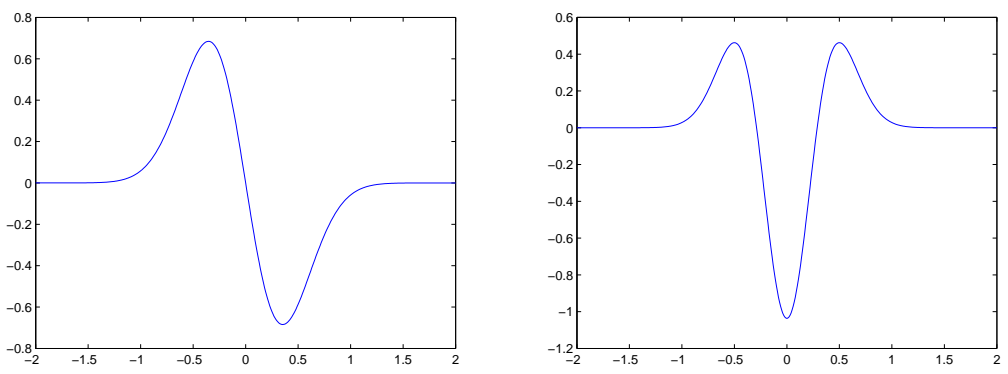

FiguRE 2. The graphs of $G_{1}^{(2)}(x)=-\sqrt{32 / \pi} x \exp \left(-4 x^{2}\right)$ (left) and $G_{2}^{(2)}(x)=\sqrt{27 /(8 \pi)}\left(12 x^{2}-1\right) \exp \left(-6 x^{2}\right)$ (right). 


\section{REFERENCES}

[1] C. de Boor, A practical guide to splines, Applied Mathematical Sciences, Vol. 27, 2001.

[2] C. de Boor, R. DeVore and A. Ron, On the Construction of Multivariate (Pre)wavelet, Constr. Approx. 9 (1993), pp. 123-166.

[3] C. de Boor, K. Höllig and S. Riemenschneider, Box Splines, Springer-Verlag, New York, 1993.

[4] J.-F. Cai, B. Dong, S. Osher and Z. Shen, Image restoration: total variation; wavelet frames; and beyond, preprint.

[5] J.-F. Cai, R.H. Chan, Z. Shen, A framelet-based image inpainting algorithm, Appl. Comput. Harmon. Anal. 24 (2008), pp. 131-149.

[6] J.-F. Cai, H. Ji, C. Liu, Z. Shen, Blind motion deblurring from a single image using sparse approximation, IEEE Conference on Computer Vision and Pattern Recognition (CVPR), Miami, 2009.

[7] J.-F. Cai, S. Osher, Z. Shen, Linearized Bregman iteration for frame based image deblurring, SIAM J. on Imaging Sciences, 2 (2009), pp. 226-252.

[8] J.-F. Cai, S. Osher, Z. Shen, Split Bregman methods and frame based image restoration, preprint, 2009.

[9] A. Chai, Z. Shen, Deconvolution: A wavelet frame approach, Numer. Math. 106 (2007), pp. 529-587.

[10] R.H. Chan, S.D. Riemenschneider, L. Shen, Z. Shen, Tight frame: an efficient way for high-resolution image reconstruction, Appl. Comput. Harmon. Anal. 17 (2004), pp. 91-115.

[11] L. H. Y. Chen, T. N. T. Goodman and S. L. Lee, Asymptotic normality of scaling functions, SIAM J. MATH. ANAL., 36 (2004), pp.323-346.

[12] I. Daubechies, Ten lectures on wavelets, SIAM Conf. Series in Appl. Math., Boston 1992.

[13] I. Daubechies, B. Han, A. Ron, Z. Shen, Framelets: MRA-based constructions of wavelet frames, Appl. Comput. Harmon. Anal. 14 (2003), pp. 1-46.

[14] B. Dong, A. C. Chien and Z. Shen, Frame based segmentation for medical images, Communications in Mathematical Sciences, 32(2010), pp. 1724-1739.

[15] B. Dong and Z. Shen, MRA-based wavelet frames and applications, IAS Lecture Notes Series, Summer Program on "The Mathematics of Image Processing", park city mathematics institute, 2010.

[16] S. J. Favier and R. A. Zalik, On the stability of frames and Riesz bases, Appl. Comput. Harmon. Anal. 2(1995), pp. 160-173.

[17] S. Goh, T. N. T. Goodman and S. L. LEE, Scale-space meets wavelets, preprint, 2011.

[18] B. Han and Z. Shen, Wavelets with short support, SIAM Journal on Mathematical Analysis, 38 (2006), pp. 530-556.

[19] R. Jia and Z. Shen, Multiresolution and Wavelets, Proc. Edinburgh Math. Soc. 37 (1994), pp.271-300.

[20] A. Ron and Z. Shen, Affine system in $L_{2}\left(\mathbb{R}^{d}\right)$ : the analyis of the analysis operator, J. Func. Anal., 148 (1997), pp. 408-447.

[21] A. Ron and Z. Shen, Gramian analysis of affine bases and affine frames, In Approximation Theory VIII-Vol2, Wavelets and Multilevel Approximation, C. K. Chui and L. L. Schumaker editors, World Scientific Publishing, New Jersey, (1995), pp.375-382.

[22] Z. Shen, Wavelet frames and image restorations, Proceedings of the International congress of Mathematicians, Vol IV, Hyderabad, India, (2010), Hindustan book Agency, (Rajendra Bhatia eds), 2834-2863.

[23] M. Unser, A. Aldroubi, and M. Eden, On the asymptotic convergence of B-splines wavelets to Gabor functions, IEEE Trans. Inf. Th., 38(1992), pp. 864-872. 
[24] Z. Xu, Multivariate splines and polytopes, J. Appr. Theory, 163 (2011), pp. 377-387.

[25] Z. Xu, Multivariate F-splines and fractional box splines, J. Four. Anal. Appl., 15 (2009), pp. 723-738.

Department of Mathematics, National University of Singapore, Block S17, 10 Lower Kent Ridge Road, Singapore, 119076

E-mail address: matzuows@nus.edu.sg

LSEC, Inst. Comp. Math., Academy of Mathematics and System Sciences, Chinese Academy of Sciences, Beijing, 100091, China

E-mail address: xuzq@lsec.cc.ac.cn 